\title{
Optimal Cheeger cuts and bisections of random geometric graphs
}

\author{
Tobias Müller ${ }^{1}$ and Mathew D. Penrose ${ }^{2}$ \\ Groningen University and University of Bath
}

April 18, 2022

\begin{abstract}
Let $d \geq 2$. The Cheeger constant of a graph is the minimum surface-tovolume ratio of all subsets of the vertex set with relative volume at most $1 / 2$. There are several ways to define surface and volume here: the simplest method is to count boundary edges (for the surface) and vertices (for the volume). We show that for a geometric (possibly weighted) graph on $n$ random points in a $d$-dimensional domain with Lipschitz boundary and with distance parameter decaying more slowly (as a function of $n$ ) than the connectivity threshold, the Cheeger constant (under several possible definitions of surface and volume), also known as conductance, suitably rescaled, converges for large $n$ to an analogous Cheeger-type constant of the domain. Previously, García Trillos et al. had shown this for $d \geq 3$ but had required an extra condition on the distance parameter when $d=2$.
\end{abstract}

\section{Introduction}

A significant recent theme in topological/geometrical data analysis and machine learning is the reconstruction of topological/geometrical properties of a continuous

\footnotetext{
${ }^{1}$ Johann Bernoulli Institute of Mathematics and Computer Science, Groningen University, P.O. box 407,9700 AK Groningen, The Netherlands: tobias.muller@rug.nl

${ }^{2}$ Department of Mathematical Sciences, University of Bath, Bath BA2 7AY, United Kingdom: m.d.penrose@bath.ac.uk

${ }^{1}$ Research partially supported by NWO grants 639.032 .529 and 612.001 .409

${ }^{2}$ Research partially supported by a STAR visitor grant, by NWO visitor grant 040.11.532, and by the Department of Mathematics at Utrecht University.

Key words and phrases: random geometric graph, Cheeger constant, conductance.

AMS Classifications: 05C80, 60D05, 62H30
} 
space such as a manifold from a random sample of points in that space via a graph, or more generally a simplicial complex, derived from the sample by connecting nearby points; see for example [8, 12, 15, 18, 21]. A prototypical graph of this type is the random geometric graph, where one connects every pair of points up to a specified distance $r$ apart (we shall consider generalization of this to allow for weighted graphs).

One quantity of considerable interest in both the continuum and discrete settings is the Cheeger constant. For a $d$-dimensional Euclidean domain $D$ (or more generally, a manifold), the Cheeger constant is the minimum perimeter-to-volume ratio of all subregions of $D$ with relative volume at most $1 / 2$ (here, when measuring the perimeter of a subregion of $D$, only the part of the boundary that is interior to $D$ is included). It can be used to provide useful bounds for the first eigenvalue gap of the Laplacian on $D$ (with Dirichlet boundary condition) [10, 7]. The analogous quantity for a graph (there are several possible definitions, as we shall describe below) similarly provides bounds for the eigengap of the graph Laplacian, and is therefore important in, among other things, the study of the mixing time of a random walk on the graph (see [2, 22] and [11, Chapter 2], for example). Cheeger constants provide a natural measure of the quality of the partition in cluster analyisis, and are important in graph-based spectral clustering methods [28].

Given the above, it is of interest mathematically, but also from the point of view of cluster analysis and machine learning, to know whether one can 'learn' about the Cheeger constant of the region $D$ from that of the random geometric graph on a sample of points in $D$. More formally, is the discrete Cheeger constant based on a geometric graph on a sample of $n$ random points with distance parameter $r_{n}$, suitably rescaled, a consistent estimator of the continuum Cheeger constant? If so, for which choices of the sequence $\left(r_{n}\right)_{n \geq 1}$ is this the case? In practical terms, one would like to use small values of $r_{n}$ to reduce the computational cost of computing the Cheeger constant of the graph, but if $r_{n}$ is too small then the graph will not be connected and its Cheeger constant will be zero. At least for regions $D$ with well-behaved boundary, the asymptotic threshold for connectivity is known to be at $r_{n}=$ const. $\times((\log n) / n)^{1 / d}[24,25]$.

Such questions were first raised and partially answered by Arias-Castro et. al. in [1]. A more complete answer was given by García Trillos et al. in [19]; they established consistency in dimensions $d \geq 3$ for all sequences $\left(r_{n}\right)$ tending to zero more slowly than the connectivity threshold, but left a gap when $d=2$, as described in the next section. In the present paper we provide an alternative proof of consistency which fills this gap. We also provide more detail than [19] for the proof in the case of some of the choices of definition of Cheeger constant of a graph.

Our argument has the potential to provide a method of showing convergence for a number of other graph optimization problems, such as those described in [26], in 
the spirit of the celebrated $\mathrm{BHH}$ result [3] for the travelling salesman problem. To illustrate this, we also give a $\mathrm{BHH}$ type result for the the minimal bisection of a random geometric graph; that is, the partition of the vertices into two equal pieces which minimises the total weight of cut edges. Finding the minimal bisection is a classic problem in computer science with applications, for example, in parallel processing and Very Large Scale Integration; see for example [4, 14].

We briefly discuss some of the sources of difficulty in these problems and the techniques used to overcome them. In the bisection problem, for example, the main difficulty is to find a good lower bound on the cost of all possible bisections of the point process. By matching the points of the sample to those of a rectangular grid with the same number of points in $D$, one may identify each such bisection with a bisection of the domain, and hence identify its cut weight with a suitably smoothed measure of the perimeter of the bisection of the domain. Using a 'liminf' Gammaconvergence bound from [17] one may then asymptotically lower bound the cost of the point process bisection by the minimal perimeter of bisections of the domain.

Loosely speaking, this is the approach of [19]. Its reliance on grid matching results means that one requires $r_{n}$ to be larger than the distances involved in the grid matching, and in $d=2$ this is known to be a stronger condition than connectivity.

Our contribution is to circumvent the need for any grid matching. To do this, we use a coarser granulation of space into boxes which are large enough for the number of points in a box to be concentrated about its mean (but which are smaller than $r_{n}$ ). We develop a local optimization technique to show that in every optimal bisection $\left(Y, Y^{c}\right)$ of the point set, each box contains mostly points of $Y$ or mostly points of $Y^{c}$ so every optimal bisection of the point process may be identified with a collection of boxes whose union bisects the domain. One may then use the Gamma-convergence techniques as before.

\section{Statement of results}

Let $d \in\{2,3, \ldots\}$ and let $D \subset \mathbb{R}^{d}$ be open. Let $\left(r_{n}\right)_{n \geq 1}$ be an $\mathbb{R}_{+}$-valued sequence, where $\mathbb{R}_{+}:=[0, \infty)$. Let $X_{1}, X_{2}, \ldots$ be a sequence of independent random $d$-vectors taking values in $D$ with common probability density function denoted $\rho$. For $n \in \mathbb{N}$ let $\mathcal{X}_{n}:=\left\{X_{1}, \ldots, X_{n}\right\}$. Given a function $\phi: \mathbb{R}_{+} \rightarrow \mathbb{R}_{+}$, let $G_{\phi}\left(\mathcal{X}_{n}, r_{n}\right)$, the $\phi$ weighted random geometric graph on the point set $\mathcal{X}_{n}$ with distance parameter $r_{n}$, be the complete graph on vertex set $\mathcal{X}_{n}$, with the weight of the edge $\{X, Y\}$ given by $\phi\left(\|Y-X\| / r_{n}\right)$ for each $X, Y \in \mathcal{X}_{n}$ with $X \neq Y$, where $\|\cdot\|$ denotes the Euclidean norm.

Two important special cases of $\phi$ are

$$
\phi_{U}(t):=\mathbf{1}_{[0,1]}(t) ; \quad \phi_{N}(t):=\exp \left(-t^{2}\right),
$$


The graph $G_{\phi_{U}}\left(\mathcal{X}_{n}\right)$ amounts to the classic Euclidean (unweighted) random geometric graph (also known as the Gilbert graph); see [24] for an overview of such graphs. The Gaussian (Normal) weight function $\phi_{N}$ is often used in spectral clustering algorithms; see for example [28]. We shall consider a general class of $\phi$ satisfying mild monotonicity and integrability conditions, which includes the two examples just mentioned.

Given $\mathcal{Y} \subset X_{n}$, set

$$
\operatorname{Cut}_{n, \phi}(\mathcal{Y}):=\sum_{y \in \mathcal{Y}} \sum_{x \in \mathcal{X}_{n} \backslash \mathcal{Y}} \phi\left(\frac{\|x-y\|}{r_{n}}\right)
$$

which is the total weight in $G_{\phi}\left(\mathcal{X}_{n}, r_{n}\right)$ of the cut edges induced by $\mathcal{Y}$, i.e. the edges from $\mathcal{Y}$ to its complement. We are interested in choosing $\mathcal{Y}$ to make $\operatorname{Cut}_{n, \phi}(\mathcal{Y})$ small but with some penalty for choices of $\mathcal{Y}$ for which $\mathcal{Y}$ or its complement is disproportionately small. This penalty takes the form of $\operatorname{dividing} \operatorname{Cut}_{n, \phi}(\mathcal{Y})$ by a 'balance term' based on the 'volume' of $\mathcal{Y}$ and its complement, where 'volume' may be measured by counting either vertices or (weighted) edges. Several choices of balance term have thus been proposed in the literature, including

$$
\operatorname{Bal}_{n, v, 1}(\mathcal{Y}):=\frac{\min \left(\operatorname{Vol}_{n, v}(\mathcal{Y}), \operatorname{Vol}_{n, v}\left(\mathcal{X}_{n} \backslash \mathcal{Y}\right)\right)}{\operatorname{Vol}_{n, v}\left(\mathcal{X}_{n}\right)}, \quad v \in\{1,2\},
$$

and

$$
\operatorname{Bal}_{n, v, 2}(\mathcal{Y}):=\frac{\operatorname{Vol}_{n, v}(\mathcal{Y}) \operatorname{Vol}_{n, v}\left(\mathcal{X}_{n} \backslash \mathcal{Y}\right)}{\left(\operatorname{Vol}_{n, v}\left(\mathcal{X}_{n}\right)\right)^{2}}, \quad v \in\{1,2\}
$$

where, with $|\mathcal{Y}|$ denoting the number of elements of $\mathcal{Y}$, we set

$$
\operatorname{Vol}_{n, 1}(\mathcal{Y}):=|\mathcal{Y}| ; \quad \operatorname{Vol}_{n, 2}(\mathcal{Y}):=\sum_{y \in \mathcal{Y}} \sum_{x \in \mathcal{X}_{n} \backslash\{y\}} \phi\left(\frac{\|x-y\|}{r_{n}}\right) .
$$

(Some authors include an extra factor of 2 in the right hand side of (2.2).) In this paper we consider the Cheeger-type functionals

$$
\mathrm{CHE}_{v, b}\left(G_{\phi}\left(\mathcal{X}_{n}, r_{n}\right)\right):=\min _{\mathcal{Y} \subset \mathcal{X}_{n}: \mathcal{Y} \neq \emptyset, \mathcal{Y} \neq \mathcal{X}_{n}}\left(\frac{\operatorname{Cut}_{n, \phi}(\mathcal{Y})}{\operatorname{Bal}_{n, v, b}(\mathcal{Y})}\right), \quad(v, b) \in\{1,2\}^{2} .
$$

The quantity being minimized in (2.3) is sometimes called the Cheeger cut of $\mathcal{Y}$ for $(v, b)=(1,1)$, the ratio cut for $(v, b)=(1,2)$, the normalized cut for $(v, b)=(2,1)$ and the sparsest cut for $(v, b)=(2,2)$; see [19] and references therein. The terms Cheeger constant and conductance with reference to a graph are used with little unanimity in the literature; all three of $\mathrm{CHE}_{1,1}\left(G_{\phi}\left(\mathcal{X}_{n}, r_{n}\right)\right), \mathrm{CHE}_{2,1}\left(G_{\phi}\left(\mathcal{X}_{n}, r_{n}\right)\right) / \operatorname{Vol}_{n, 2}\left(\mathcal{X}_{n}\right)$ 
and $\mathrm{CHE}_{2,2}\left(G_{\phi}\left(\mathcal{X}_{n}, r_{n}\right)\right) /\left(\operatorname{Vol}_{n, 2}\left(\mathcal{X}_{n}\right)\right)^{2}$ could be called the Cheeger constant or conductance of the graph $G_{\phi}\left(\mathcal{X}_{n}, r_{n}\right)$; see for example [19], [1], [2], [22].

One may also consider continuum analogues. Let $\mathcal{B}(D)$ denote the Borel $\sigma$ field on $D$. Let $\nu$ be the measure on $(D, \mathcal{B}(D))$ with Lebesgue density $\rho$ (i.e., the distribution of $\left.X_{1}\right)$. For $u \in L^{1}(\nu)$ set

$$
T V(u):=\sup \left\{\int_{D} u(x) \operatorname{div}(\Phi)(x) d x: \Phi \in C_{c}^{1}\left(D: \mathbb{R}^{d}\right),|\Phi(x)| \leq \rho^{2}(x) \forall x \in D\right\} .
$$

Here $C_{c}^{1}\left(D: \mathbb{R}^{d}\right)$ denotes the class of all continuously differentiable functions from $D$ to $\mathbb{R}^{d}$ having support that is compact and contained in $D$. For $\Phi=\left(\Phi_{1}, \ldots, \Phi_{d}\right) \in$ $C_{c}^{1}\left(D: \mathbb{R}^{d}\right)$, and $x=\left(x_{1}, \ldots, x_{d}\right) \in D$, we define $\operatorname{div}(\Phi)(x)=\left.\sum_{i=1}^{d} \frac{\partial \Phi_{i}}{\partial x_{i}}\right|_{x}$. We shall assume throughout that $D$ is bounded and connected, and that $D$ has Lipschitz boundary, which means that each $x \in \partial D$ (the boundary of $D$ ) has a neighbourhood $U$ such that the restriction of $\partial D$ to $U$ is the graph of a Lipschitz function after a suitable rotation. We shall also assume that the density $\rho: D \rightarrow \mathbb{R}_{+}$is continuous with $\rho_{\max }:=\sup _{x \in D} \rho(x)<\infty$ and $\rho_{\min }:=\inf _{x \in D} \rho(x)>0$. Then, according to (3.3) of [17] (see also Proposition 2.33 of [20]), if $u=\mathbf{1}_{A}$ is the indicator function of a set $A \subset \mathbb{R}^{d}$ with $C^{1}$ boundary (defined analogously to the Lipschitz boundary above), then

$$
T V\left(\mathbf{1}_{A}\right)=\int_{\partial A \cap D} \rho^{2}(x) \mathcal{H}^{d-1}(d x)
$$

where $\mathcal{H}^{d-1}$ is the $(d-1)$-dimensional Hausdorff measure. More generally than (2.4), we assert for all $A \in \mathcal{B}(D)$ with $T V\left(\mathbf{1}_{A}\right)<\infty$ that

$$
T V\left(\mathbf{1}_{A}\right)=\int_{\partial^{*} A \cap D} \rho^{2}(x) \mathcal{H}^{d-1}(d x),
$$

where $\partial^{*} A$ is the De Giorgi reduced boundary of $A$ (see [6, Definition 1.54]). This assertion follows from (3.5) of [19] and Theorem 1.55 of [6]. We define the continuum Cheeger functionals of $(D, \rho)$ by

$$
\mathrm{CHE}_{v, b}(D, \rho):=\inf _{A \in \mathcal{B}(D): 0<\nu(A)<1}\left(\frac{T V\left(\mathbf{1}_{A}\right)}{\operatorname{Bal}_{\nu, v, b}(A)}\right), \quad(v, b) \in\{1,2\}^{2},
$$

where for $A \in \mathcal{B}(D)$ we set

$$
\begin{gathered}
\operatorname{Bal}_{\nu, v, 1}(A):=\frac{\min \left(\operatorname{Vol}_{\nu, v}(A), 1-\operatorname{Vol}_{\nu, v}(D \backslash A)\right)}{\operatorname{Vol}_{\nu, v}(D)} ; \\
\operatorname{Bal}_{\nu, v, 2}(A):=\frac{\operatorname{Vol}_{\nu, v}(A) \operatorname{Vol}_{\nu, v}(D \backslash A)}{\left(\operatorname{Vol}_{\nu, v}(D)\right)^{2}}
\end{gathered}
$$


with

$$
\operatorname{Vol}_{\nu, v}(A):=\int_{A}(\rho(x))^{v} d x, \quad v \in\{1,2\} .
$$

Using (2.4), it is easy to see for $(v, b) \in\{1,2\}^{2}$ that $\mathrm{CHE}_{v, b}(D, \rho)<\infty$. It is less easy to see that $\mathrm{CHE}_{v, b}(D, \rho)>0$ in general (but it is stated in the MathSciNet review of [10] that this is well-known in the case where $\rho$ is constant, from which we can deduce the same for general $\rho$ ), but we shall not use this.

It may be the case that in some circumstances, the definition of $\mathrm{CHE}_{v, b}(D, \rho)$ is unaffected by restricting the minimum to sets $A$ with smooth boundary, for which we can use the definition (2.4) of $T V\left(\mathbf{1}_{A}\right)$. A result along these lines (for constant $\rho$ and under a further smoothness condition on $\partial D$ ) appears in [9], but to give such a result in the generality considered here would be beyond the scope of the present paper.

We shall assume $\phi$ satisfies the following conditions:

$$
\begin{array}{r}
\phi(r) \geq \phi(s) \quad \forall r, s \in \mathbb{R}_{+} \text {with } r \leq s ; \\
\phi(0)>0 \text { and } \phi \text { is continuous on }[0, \delta] \text { for some } \delta>0 ; \\
\sigma_{\phi}:=\int_{\mathbb{R}^{d}} \phi(\|x\|)\left|x_{1}\right| d x<\infty
\end{array}
$$

where here $x_{1}$ denotes the first co-ordinate of $x$. The quantity $\sigma_{\phi}$ is sometimes called the 'surface tension' of $\phi$. In particular $\sigma_{\phi_{U}}$ is twice the quantity denoted $\gamma_{d}$ in eqn (4) of [1]. We have

$$
\sigma_{\phi_{U}}=\frac{2 \pi^{(d-1) / 2}}{(d+1) \Gamma((d+1) / 2)} ; \quad \sigma_{\phi_{N}}=\pi^{(d-1) / 2} .
$$

The first identity of (2.9) is derived in [1], and the second is standard. For any two $\mathbb{R}_{+}$-valued sequences $\left(a_{n}\right)_{n \geq 1}$ and $\left(b_{n}\right)_{n \geq 1}$ we write $a_{n} \gg b_{n}$ or $b_{n} \ll a_{n}$ or $b_{n}=o\left(a_{n}\right)$ if $\lim _{n \rightarrow \infty}\left(b_{n} / a_{n}\right)=0$ (defining $0 / 0:=1$ ). We also sometimes write $b_{n}=O\left(a_{n}\right)$ if $\lim \sup _{n \rightarrow \infty}\left(b_{n} / a_{n}\right)<\infty$, and write $b_{n}=\Theta\left(a_{n}\right)$ if both $b_{n}=O\left(a_{n}\right)$ and $a_{n}=O\left(b_{n}\right)$. We use the term 'almost surely' (or 'a.s.') to denote 'with probabilty one' (rather than 'with probability tending to one'). The following is our main result.

Theorem 2.1. Suppose that $D$ is a nonempty, open, bounded, connected subset of $\mathbb{R}^{d}$ with Lipschitz boundary, and $\rho: D \rightarrow \mathbb{R}_{+}$is a continuous probability density function satisfying $\rho_{\max }<\infty$ and $\rho_{\min }>0$. Suppose that $\phi$ satisfies (2.6)-(2.8), and that $\left(r_{n}\right)_{n \geq 1}$ satisfies $n r_{n}^{d} \gg \log n$ and $r_{n} \ll 1$. Let $(v, b) \in\{1,2\}^{2}$. Then

$$
\lim _{n \rightarrow \infty}\left(\frac{\mathrm{CHE}_{v, b}\left(G_{\phi}\left(\mathcal{X}_{n}, r_{n}\right)\right)}{n^{2} r_{n}^{d+1}}\right)=\left(\sigma_{\phi} / 2\right) \operatorname{CHE}_{v, b}(D, \rho), \quad \text { a.s. }
$$


This was already shown by García Trillos et al. in [19, Theorem 9], except that in the case $d=2$ they require the extra condition that $n r_{n}^{2} \gg(\log n)^{3 / 2}$; our result answers a question raised in Remark 2 of [19] as to whether we can do without this extra condition (yes we can). Moreover, in [19] the proof is provided only for the case $v=1$. Previously Arias-Castro et al. [1] asked about the limiting behaviour when $(v, b)=(2,1)$. To relate the case $(v, b)=(2,1)$ of the above result to the limiting behaviour of the Cheeger constant as defined in [1, note that as a special case of Lemma 4.2 below we have

$$
\lim _{n \rightarrow \infty}\left(n^{2} r_{n}^{d}\right)^{-1} \operatorname{Vol}_{n, 2}\left(\mathcal{X}_{n}\right)=\int_{D} \rho(x)^{2} d x \int_{\mathbb{R}^{d}} \phi(\|y\|) d y .
$$

The case $\phi=\phi_{U}$ of (2.11) was proved in Theorem 3.17 of [24]. Note that the right hand side of (2.11) is finite by (2.8) and the assumptions on $D$ and $\rho$.

Our next theorem shows that under the same hypotheses as in Theorem 2.1, the empirical measure of the optimising choice of $\mathcal{Y}$ in (2.3) converges subsequentially to the restriction of $\nu$ to an optimising set in the definition (2.5). We use the standard notion of weak convergence of probability measures on a metric space, as described in [5], for example. Given $A \in \mathcal{B}(D)$, let $\left.\nu\right|_{A}$ denote the restriction of the measure $\nu$ to $A$, i.e. the measure on $D$ with density $\rho(\cdot) \mathbf{1}_{A}(\cdot)$.

Theorem 2.2. Suppose the hypotheses of Theorem 2.1 hold. Almost surely, for any sequence of minimisers $\mathcal{Y}_{n}$ in the definition (2.3) of $\mathrm{CHE}_{v, b}\left(G_{\phi}\left(\mathcal{X}_{n}, r_{n}\right)\right)$ and any infinite $\mathcal{N} \subset \mathbb{N}$, there exists an infinite $\mathcal{N}^{\prime} \subset \mathcal{N}$ and a minimising set $A$ in the definition (2.5), such that we have the weak convergence of measures

$$
\left.\sum_{y \in \mathcal{Y}_{n}} n^{-1} \delta_{y} \rightarrow \nu\right|_{A} \quad \text { as } n \rightarrow \infty \text { through } \mathcal{N}^{\prime} .
$$

When the minimising set $A$ is essentially unique up to complementation, one can re-phrase the preceding result without needing to take subsequences, as follows.

Corollary 2.3. Suppose that the hypotheses of Theorem 2.1 hold, and also that the minimising set $A$ in the definition (2.5) of $\mathrm{CHE}_{v, b}(D, \rho)$ is unique, up to complementation and adding or removing sets of $(d-1)$-dimensional measure zero.

Then, almost surely, for any sequence of minimisers $\mathcal{Y}_{n}$ in the definition (2.3) of $\mathrm{CHE}_{v, b}\left(G_{\phi}\left(\mathcal{X}_{n}, r_{n}\right)\right)$ there exists a sequence $(j(n), n \in \mathbb{N})$ taking values in $\{0,1\}$, such that setting $\overline{\mathcal{Y}}_{n}=\mathcal{Y}_{n}$ if $j(n)=1$ and $\overline{\mathcal{Y}}_{n}=\mathcal{X}_{n} \backslash \mathcal{Y}_{n}$ if $j(n)=0$, we have

$$
\left.\sum_{y \in \overline{\mathcal{Y}}_{n}} n^{-1} \delta_{y} \rightarrow \nu\right|_{A} \quad \text { as } n \rightarrow \infty .
$$


For completeness, we shall provide a proof of Corollary 2.3 at the end of Section

The Prohorov distance on probability measures on $D$ is a metrization of weak convergence (see [5]). Another interpretation of Theorem 2.2 is that, almost surely, for any sequence of minimisers $\mathcal{Y}_{n}$ the Prohorov distance from $\sum_{y \in \mathcal{Y}_{n}} n^{-1} \delta_{y}$ to the set of measures of the form $\nu_{A}$ with $A$ a minimising set in the definition (2.5) of $\mathrm{CHE}_{v, b}(D, \rho)$, tends to zero.

A result resembling Theorem 2.2 is provided in [19, Theorem 9], but again under the extra condition $n r_{n}^{2} \gg(\log n)^{3 / 2}$ when $d=2$, and again with proofs given only for $v=1$. Also, we use a different (and apparently simpler) notion of weak convergence of measures than the one used there. Both of these distinctions are related to the fact that the proof in [19] proceeds via certain transportations of measures (we discuss this further below).

Next we describe a similar result for the minimum bisection functional

$$
\operatorname{MBIS}\left(G_{\phi}\left(\mathcal{X}_{n}, r_{n}\right)\right):=\min \left\{\mathrm{Cut}_{n, \phi}(\mathcal{Y}): \mathcal{Y} \subset \mathcal{X}_{n},|\mathcal{Y}|=\lfloor n / 2\rfloor\right\} .
$$

This functional has been considered in [13, 24] and elsewhere. For the regime considered here with $n r_{n}^{d} \gg \log n$ (in fact for a greater range of regimes for $\left(r_{n}\right)$ ), it was shown in [26] that for for $\phi=\phi_{U}$ under the additional assumption that $D$ is the unit cube and $\nu$ is the uniform distribution on $D$ that $\operatorname{MBIS}\left(G_{\phi_{U}}\left(\mathcal{X}_{n}, r_{n}\right)\right)=$ $\Theta\left(n^{2} r_{n}^{d+1}\right)$, almost surely. Under the further assumption that $d=2$ and using the $\ell_{\infty}$ distance to define the random geometric graph, explicit upper and lower bounds are given in [13] for the limits superior and inferior of $\operatorname{MBIS}\left(G_{\phi_{U}}\left(\mathcal{X}_{n}, r_{n}\right)\right) /\left(n^{2} r_{n}^{d+1}\right)$ which differ by a factor of 4 . In this section we give a BHH-type result for this problem (for general $d$ and $D$, using the Euclidean distance), i.e. a strong law for $\operatorname{MBIS}\left(G_{\phi}\left(\mathcal{X}_{n}, r_{n}\right)\right)$ in the regime $n r_{n}^{d} \gg \log n$. The result goes as follows.

Theorem 2.4. Suppose the hypotheses of Theorem 2.1 hold. Then

$$
\lim _{n \rightarrow \infty}\left(\frac{\operatorname{MBIS}\left(G_{\phi}\left(\mathcal{X}_{n}, r_{n}\right)\right)}{n^{2} r_{n}^{d+1}}\right)=\left(\sigma_{\phi} / 2\right) \operatorname{MBIS}_{\nu}(D), \quad \text { a.s. }
$$

where we set

$$
\operatorname{MBIS}_{\nu}(D):=\inf _{A \in \mathcal{B}(D): \nu(A)=1 / 2} T V\left(\mathbf{1}_{A}\right) .
$$

One might also consider these problems for geometric graphs on other sequences of point process besides the binomial point process $\mathcal{X}_{n}$. For example, the results should carry through if instead of $\mathcal{X}_{n}$ one considered a Poisson point process $\mathcal{X}_{N_{n}}$ with $N_{n}$ Poisson $(n)$ distributed and independent of $\left(X_{1}, X_{2}, \ldots\right)$. They should carry through because the main probabilistic tools used are the Chernoff bounds (3.10) 
and (3.11) for the binomial distribution, and analogous bounds are also available for the Poisson distribution.

Another possibility would be to consider instead of $\mathcal{X}_{n}$ a deterministic rectilinear grid with spacings of size $n^{-1 / d}$. In this case one might be able to get the same results with the condition $n r_{n}^{d} \gg \log n$ weakened to $n r_{n}^{d} \gg 1$.

Another natural extension of the results would be to consider Riemannian manifolds, which is the setting of the original work of Cheeger [10]. We have not attempted this but it seems likely that our methods can be extended to the manifold setting. One reason to include non-uniform $\rho$ in our results is that this may be useful in extending them the manifold setting.

For the rest of this paper we assume that $D, \rho$ and $\phi$ satisfy the conditions assumed in the statement of Theorem 2.1. We also assume that $r_{n} \ll 1$, and that $n r_{n}^{d} \gg \log n$.

Here is an overview of our method of proof of the 'liminf' part of (2.10) and (2.12). We divide $D$ into boxes of side $\gamma_{n} r_{n}$, where $\gamma_{n} \rightarrow 0$ slowly. By Chernoff bounds (Lemma 3.2), the number of points in each box is close to its expected value. Given an optimal subset $\mathcal{Y} \subset \mathcal{X}_{n}$, we adjust $\mathcal{Y}$ to a set $\mathcal{Y}^{\prime} \subset \mathcal{X}_{n}$ that is not too different from $\mathcal{Y}$, such that all boxes have mostly points in $\mathcal{Y}^{\prime}$ or mostly points in $\mathcal{X}_{n} \backslash \mathcal{Y}^{\prime}$, and which is also close to optimal. Then we approximate to $\mathcal{Y}$ by the union of boxes containing mostly points of $\mathcal{Y}^{\prime}$ and estimate the discrete cut of $\mathcal{Y}$ by an approximation to the perimeter for this union of boxes. We then use a Gamma-convergence result from [17] (Lemma 3.3 below) to derive the desired liminf inequality.

The method of [19] is related, but relies (via the paper [17]) on results of Shor et al. [23, 27, extended in [16] to the class of domains considered here, on the existence of a matching of a grid of side $\Theta\left(n^{-1 / d}\right)$ to the random point set $\mathcal{X}_{n}$, with maximum displacement at most $O\left(((\log n) / n)^{1 / d}\right)$ (for $\left.d \geq 3\right)$ or $O\left(\left((\log n)^{3 / 2} / n\right)^{1 / d}\right)$ (for $d=$ 2 ). (It also requires a notion of weak convergence of pairs $\left(\mu_{n}, T_{n}\right)$ where $\mu_{n}$ is a measure and $T_{n}$ a functional.) As mentioned earlier, our method avoids relying on grid matchings enabling us, when $d=2$, to relax the condition $r_{n} \gg(\log n)^{3 / 4} n^{-1 / 2}$ (required in [19]) to $r_{n} \gg(\log n)^{1 / 2} n^{-1 / 2}$ ).

We have included the cases with $v=2$ in our proofs. This entails extra work; see for example Lemmas 4.3 and 5.6. We suspect that a similar amount of work would be needed to fill in the details of proof for $v=2$ of the corresponding results in [19]. 


\section{Preliminaries}

Since we assume that $n r_{n}^{d} \gg \log n$ and $r_{n} \ll 1$, we can and do choose a sequence $\left(\gamma_{n}\right)_{n \geq 1}$ of constants, such that $\gamma_{n} \ll 1$ and also

$$
n \gamma_{n}^{d+2} r_{n}^{d} \gg \log n ; \quad \quad \gamma_{n}^{d+4} \gg r_{n}
$$

In other words the $\gamma_{n}$ tend to zero, but possibly very slowly.

Given $n \in \mathbb{N}$, divide $\mathbb{R}^{d}$ into half-open rectilinear cubes $Q_{1, n}^{\prime}, Q_{2, n}^{\prime}, \ldots$ of side $\gamma_{n} r_{n}$, with the centre of $Q_{i, n}^{\prime}$ denoted $z_{i, n}$. To be definite, assume the origin is one of the points $z_{i, n}$. Let $S_{n}:=\left\{i \in \mathbb{N}: Q_{i, n}^{\prime} \subset D\right\}$ (which is a nonempty set for large enough $n$ ), and let $D_{n}:=\left\{z_{i, n}: i \in S_{n}\right\}$.

Suppose $n$ is such that $S_{n} \neq \emptyset$. For $j \in \mathbb{N}$, let $i=I(j, n) \in S_{n}$ be chosen so that $z_{i, n}$ is the nearest point of $D_{n}$ to $z_{j, n}$, using the lexicographic ordering on $\mathbb{R}^{d}$ to break any ties. (In particular, if $j \in S_{n}$ then $I(j, n)=j$.) Then for each $i \in S_{n}$, define the set

$$
Q_{i, n}:=\cup_{\{j: i=I(j, n)\}}\left(Q_{j, n}^{\prime} \cap D\right) .
$$

That is, $Q_{i, n}$ is the union of $Q_{i, n}^{\prime}$ itself, and those boundary cubes $Q_{j, n}^{\prime}$ which have $Q_{i, n}^{\prime}$ as the nearest interior cube (intersected with $D$ ). We shall refer to the sets $Q_{i, n}, i \in S_{n}$ as boxes, even though only those sets $Q_{i, n}$ lying away from the boundary of $D$ are necessarily cubes.

Since $D$ has Lipschitz boundary, using a compactness argument we can find constants $C \geq d$ and $n_{0} \in \mathbb{N}$ such that for all $n \geq n_{0}$, for all boundary boxes $Q_{j, n}$ there is an interior box within distance $(C / 3) \gamma_{n}$, and hence

$$
\|x-y\| \leq C \gamma_{n} r_{n}, \quad \forall i \in S_{n}, x, y \in Q_{i, n} .
$$

For each $n \in \mathbb{N}$ we define a function $\phi_{n}(x, y)$ (respectively $\phi^{(n)}(x, y)$ ) that approximate the weight function $\phi\left(\|x-y\| / r_{n}\right)$ from below (respectively, from above) and is constant on each product of boxes, as follows: for each $i, j \in S_{n}$ set

$$
\begin{aligned}
\phi_{n}(x, y) & :=\inf _{x^{\prime} \in Q_{i, n}, y^{\prime} \in Q_{j, n}} \phi\left(\left\|x^{\prime}-y^{\prime}\right\| / r_{n}\right), \quad x \in Q_{i, n}, y \in Q_{j, n} \\
\phi^{(n)}(x, y) & :=\sup _{x^{\prime} \in Q_{i, n}, y^{\prime} \in Q_{j, n}} \phi\left(\left\|x^{\prime}-y^{\prime}\right\| / r_{n}\right), \quad x \in Q_{i, n}, y \in Q_{j, n} .
\end{aligned}
$$

Lemma 3.1. There exist constants $C^{\prime} \in(0, \infty), n_{1} \geq n_{0}$, and $\left(\tilde{\gamma}_{n}\right)_{n \in \mathbb{N}}$ with $\tilde{\gamma}_{n} \ll 1$, depending only on $D$ and $\phi$, such that for all $n \geq n_{1}$ and all $x, y \in D$ we have

$$
\phi\left(\|x-y\| / r_{n}\right) \geq \phi_{n}(x, y) \geq\left(1-\tilde{\gamma}_{n}\right) \phi\left(\|x-y\| / \tilde{r}_{n}\right)
$$


and

$$
\phi\left(\|x-y\| / r_{n}\right) \leq \phi^{(n)}(x, y) \leq\left(1+\tilde{\gamma}_{n}\right) \phi\left(\|x-y\| / r_{n}^{\prime}\right),
$$

where we set $\tilde{r}_{n}:=\left(1-C^{\prime} \gamma_{n}\right) r_{n}$ and $r_{n}^{\prime}:=\left(1+C^{\prime} \gamma_{n}\right) r_{n}$.

Proof. The first inequality of (3.6) is clear from the definition (3.4). To prove the second inequality, observe that for any $i, j \in S_{n}$, for $x, x^{\prime} \in Q_{i, n}$ and $y, y^{\prime} \in Q_{j, n}$, by (3.3) we have $\left\|y^{\prime}-x^{\prime}\right\| \leq\|y-x\|+2 C \gamma_{n} r_{n}$, so that using (2.6) we have

$$
\phi_{n}(x, y) \geq \phi\left(\frac{\|x-y\|}{r_{n}}+2 C \gamma_{n}\right) \text {. }
$$

Using assumption (2.7), choose $a>0$ with $\phi$ continuous (and hence uniformly continuous) on the interval $[0,2 a]$ and $\phi(2 a)>0$. Then by the uniform continuity, there is a function $h: \mathbb{R}_{+} \rightarrow \mathbb{R}_{+}$with $h(u) \rightarrow 0$ as $u \downarrow 0$ such that for all $t, u \in[0, a]$ we have $\phi(t+u) \geq \phi(t)-h(u)$.

For $0 \leq t \leq a$, and for $n$ large enough so that $\gamma_{n} \leq a /(2 C)$, we have that

$$
\frac{\phi\left(t+2 C \gamma_{n}\right)}{\phi(t)} \geq \frac{\phi(t)-h\left(2 C \gamma_{n}\right)}{\phi(t)} \geq 1-\frac{h\left(2 C \gamma_{n}\right)}{\phi(2 a)}
$$

Setting $\tilde{\gamma}_{n}:=h\left(2 C \gamma_{n}\right) / \phi(2 a)$, by (3.8) we have for $\|x-y\| \leq a r_{n}$ that

$$
\phi_{n}(x, y) \geq\left(1-\tilde{\gamma}_{n}\right) \phi\left(\frac{\|x-y\|}{r_{n}}\right) \geq\left(1-\tilde{\gamma}_{n}\right) \phi\left(\frac{\|x-y\|}{\tilde{r}_{n}}\right) .
$$

Take $C^{\prime}>2 C / a$. Then for $t>a r_{n}$, if $n$ is large enough so that $C^{\prime} \gamma_{n}<1$ we have

$$
\frac{t}{\left(1-C^{\prime} \gamma_{n}\right) r_{n}}>\left(t / r_{n}\right)\left(1+C^{\prime} \gamma_{n}\right)>\left(t / r_{n}\right)+a C^{\prime} \gamma_{n}>\left(t / r_{n}\right)+2 C \gamma_{n}
$$

Hence by (3.8), for $\|x-y\|>a r_{n}$ we have

$$
\phi_{n}(x, y) \geq \phi\left(\|x-y\| / \tilde{r}_{n}\right) \geq\left(1-\tilde{\gamma}_{n}\right) \phi\left(\|x-y\| / \tilde{r}_{n}\right) .
$$

Combined with (3.9) for $\|x-y\| \leq a r_{n}$, this gives us (3.6).

The proof of (3.7) is similar.

For $n \in \mathbb{N}$ and $p \in[0,1]$ let $\operatorname{Bi}(n, p)$ denote a binomial random variable with parameters $n, p$. Also let $H(x)=1-x+x \log x$ for $x>0$, and let $H(0)=1$. The following Chernoff-type bounds are well-known (see for example Lemma 1.1 of [24]):

$$
\begin{aligned}
& \mathbb{P}[\operatorname{Bi}(n, p) \geq k] \leq \exp \left(-n p H\left(\frac{k}{n p}\right)\right), \quad k \geq n p ; \\
& \mathbb{P}[\operatorname{Bi}(n, p) \leq k] \leq \exp \left(-n p H\left(\frac{k}{n p}\right)\right), \quad k \leq n p .
\end{aligned}
$$


Lemma 3.2. There exists an almost surely finite random variable $N$ such that for all $n \geq N$ and all $i \in S_{n}$ we have

$$
\left|\mathcal{X}_{n} \cap Q_{i, n}\right| \leq\left(1+\gamma_{n}\right) n \nu\left(Q_{i, n}\right)
$$

and

$$
\left|\mathcal{X}_{n} \cap Q_{i, n}\right| \geq\left(1-\gamma_{n}\right) n \nu\left(Q_{i, n}\right) .
$$

Proof. By Taylor's theorem, for $x \in \mathbb{R}$ with $\|x\|$ sufficiently small we have that $H(1+x)>(1 / 3) x^{2}$, and hence for large enough $n$ we have for all $i \in S_{n}$ by (3.10) that

$$
\begin{array}{r}
\mathbb{P}\left[\left|\mathcal{X}_{n} \cap Q_{i, n}\right|>\left(1+\gamma_{n}\right) n \nu\left(Q_{i, n}\right)\right] \leq \exp \left(-n \nu\left(Q_{i, n}\right) H\left(1+\gamma_{n}\right)\right) \\
\leq \exp \left(-n \rho_{\min } r_{n}^{d} \gamma_{n}^{d+2} / 3\right)
\end{array}
$$

and by (3.1) this bound is $O\left(n^{-9}\right)$. Since $D$ is bounded and $n\left(\gamma_{n} r_{n}\right)^{d} \rightarrow \infty$ by (3.1), we have that $\left|S_{n}\right|=O(n)$. Therefore it follows by (3.14), the union bound and the Borel-Cantelli lemma that (3.12) holds for all but finitely many $n$, almost surely. The proof of (3.13) is similar, this time using (3.11).

We shall repeatedly use the following result due to García Trillos and Slepčev [17. Given $r>0$ define the functional $T V_{\phi, r}$ on $L^{1}(\nu)$ by

$$
T V_{\phi, r}(u):=r^{-d-1} \int_{D} \int_{D} \phi\left(\frac{\|x-y\|}{r}\right)|u(x)-u(y)| \nu(d x) \nu(d y),
$$

as in (1.9) on page 203 of [17] (see page 195 of [17] for the definition of $\phi_{r}$ used there). Note that $T V_{\phi, r}(a u)=a T V_{\phi, r}(u)$ for all $a>0$. For $u$ of the form $u=\mathbf{1}_{A}$ for some $A \in \mathcal{B}(D)$, the functional $T V_{\phi, r}(u)$ may be viewed as providing a smoothed measure of the perimeter of $A$.

Lemma 3.3. Let $\left(\varepsilon_{n}\right)_{n \geq 1}$ be a $(0,1)$-valued sequence with $\varepsilon_{n} \ll 1$. Then:

(i) [liminf lower bound] for any $L^{1}(\nu)$-valued sequence $\left(u_{n}\right)_{n \geq 1}$ converging in $L^{1}(\nu)$ to some $u \in L^{1}(\nu)$, we have

$$
\liminf _{n \rightarrow \infty} T V_{\phi, \varepsilon_{n}}\left(u_{n}\right) \geq \sigma_{\phi} T V(u)
$$

(ii) For any $u \in L^{1}(\nu)$,

$$
\lim _{n \rightarrow \infty} T V_{\phi, \varepsilon_{n}}(u)=\sigma_{\phi} T V(u)
$$

(iii) [Compactness] If $\left(u_{n}\right)_{n \geq 1}$ is an $L^{1}(\nu)$-valued sequence that is bounded in $L^{1}(\nu)$, and $T V_{\phi, \varepsilon_{n}}\left(u_{n}\right)$ is bounded, then there is a subsequence $\left(n_{k}\right)$ along which $u_{n_{k}} \rightarrow u$ in $L^{1}(\nu)$ for some $u \in L^{1}(\nu)$. 
Proof. Part (i) is from Theorem 4.1 of [17] and the definition of Gamma-convergence (also in [17], for example). Part (ii) is from Remark 4.3 of [17]. Part (iii) is also from Theorem 4.1 of [17].

For the reader's convenience, we offer the following clarifications to [17], kindly provided by Nicolás García Trillos. In (4.9) of that paper, the constant $C$ needs to be allowed to depend on $\delta$ but this does not affect the subsequent argument there. Also, the first display of page 230 of [17] is incorrect; one can avoid needing to use this display by changing $\eta_{\varepsilon}$ to $\eta_{\varepsilon / 4}$ in (4.22) of [17] and then changing $\eta_{\varepsilon}$ to $\eta_{\varepsilon / 4}$ and $\eta_{4 \varepsilon}$ to $\eta_{\varepsilon}$ throughout page 229 of [17].

\section{Upper bound}

Throughout this section we assume $D, \rho, \phi$ and $\left(r_{n}\right)_{n \geq 1}$ satisfy the assumptions of Theorem 2.1. We prove the following result, which is the easier half of Theorem 2.1.

Proposition 4.1. Given $(v, b) \in\{1,2\}^{2}$, we have

$$
\limsup _{n \rightarrow \infty}\left(\frac{\mathrm{CHE}_{v, b}\left(G_{\phi}\left(\mathcal{X}_{n}, r_{n}\right)\right)}{n^{2} r_{n}^{d+1}}\right) \leq\left(\sigma_{\phi} / 2\right) \operatorname{CHE}_{v, b}(D, \rho) .
$$

The proof of this in the case $v=2$ requires the following result which also justifies our earlier assertion (2.11):

Lemma 4.2. Let $A \in \mathcal{B}(D)$. Then, almost surely,

$$
\lim _{n \rightarrow \infty}\left(n^{2} r_{n}^{d}\right)^{-1} \operatorname{Vol}_{n, 2}\left(\mathcal{X}_{n} \cap A\right)=\int_{A} \rho(x)^{2} d x \int_{\mathbb{R}^{d}} \phi(\|y\|) d y
$$

Moreover, there exists a constant $C^{\prime \prime} \in(0, \infty)$ such that a.s., for all large enough $n$ and all $\mathcal{Y} \subset \mathcal{X}_{n}$,

$$
\left(C^{\prime \prime}\right)^{-1} n r_{n}^{d}|\mathcal{Y}| \leq \operatorname{Vol}_{n, 2}(\mathcal{Y}) \leq C^{\prime \prime} n r_{n}^{d}|\mathcal{Y}|
$$

Proof. Let $N$ be as in Lemma 3.2. Then for all $n \geq N$, using the first inequality of (3.7), then (3.12) followed by the second inequality of (3.7), we have for all $X \in \mathcal{X}_{n}$ that

$$
\begin{aligned}
\operatorname{Vol}_{n, 2}(\{X\}) & \leq\left(1+\gamma_{n}\right) n \int \phi^{(n)}(X, y) \nu(d y) \\
& \leq\left(1+\gamma_{n}\right)\left(1+\tilde{\gamma}_{n}\right) n \int \phi\left(\frac{\|y-X\|}{r_{n}^{\prime}}\right) \nu(d y) .
\end{aligned}
$$


Also, using (3.6) and (3.13) we have for all $n$ large enough so that $n \geq N$ and $\gamma_{n} n \nu\left(Q_{i, n}\right) \geq 1$ for all $i \in S_{n}$, and all $X \in \mathcal{X}_{n}$, that

$$
\begin{aligned}
\operatorname{Vol}_{n, 2}(\{X\}) & \geq\left(1-2 \gamma_{n}\right) n \int \phi_{n}(X, y) \nu(d y) \\
& \geq\left(1-2 \gamma_{n}\right)\left(1-\tilde{\gamma}_{n}\right) n \int \phi\left(\frac{\|y-X\|}{\tilde{r}_{n}}\right) \nu(d y) .
\end{aligned}
$$

Defining $\rho(\cdot) \equiv 0$ on $\mathbb{R}^{d} \backslash D$ and recalling the definition of $r_{n}^{\prime}$ from Lemma 3.1, for $x \in D$ let us set

$$
h_{n}(x):=r_{n}^{-d} \int_{\mathbb{R}^{d}} \phi\left(\frac{\|y-x\|}{r_{n}^{\prime}}\right) \rho(y) d y=\left(\frac{r_{n}^{\prime}}{r_{n}}\right)^{d} \int_{\mathbb{R}^{d}} \phi(\|u\|) \rho\left(x+r_{n}^{\prime} u\right) d u .
$$

By (4.4), it is almost surely the case that for large enough $n$ we have

$$
\operatorname{Vol}_{n, 2}\left(\mathcal{X}_{n} \cap A\right) \leq\left(1+\tilde{\gamma}_{n}\right)\left(1+\gamma_{n}\right) n \sum_{X \in \mathcal{X}_{n} \cap A} r_{n}^{d} h_{n}(X) .
$$

Since $\rho$ is continuous on $D, \rho_{\max }<\infty$ and $I_{\phi}:=\int_{\mathbb{R}^{d}} \phi(\|x\|) d x$ is finite by (2.8), by dominated convergence we have $h_{n}(x) \rightarrow \rho(x) I_{\phi}=: h(x)$ for all $x \in D$. Moreover $h_{n}(x)$ is bounded uniformly in $(n, x)$. Therefore by a version of the strong law of large numbers,

$$
\lim _{n \rightarrow \infty} n^{-1} \sum_{i=1}^{n} h_{n}\left(X_{i}\right) \mathbf{1}_{A}\left(X_{i}\right)=\mathbb{E}\left[h\left(X_{1}\right) \mathbf{1}_{A}\left(X_{1}\right)\right]=I_{\phi} \int_{A} \rho(x)^{2} d x .
$$

Hence by (4.7) we have

$$
\limsup _{n \rightarrow \infty} n^{-2} r_{n}^{-d} \operatorname{Vol}_{n, 2}\left(\mathcal{X}_{n} \cap A\right) \leq I_{\phi} \int_{A} \rho(x)^{2} d x .
$$

A similar argument using (4.5) instead of (4.4) shows that

$$
\liminf _{n \rightarrow \infty} n^{-2} r_{n}^{-d} \operatorname{Vol}_{n, 2}\left(\mathcal{X}_{n} \cap A\right) \geq I_{\phi} \int_{A} \rho(x)^{2} d x
$$

and together with (4.8) this gives us (4.2) as asserted.

Finally, observe that the proof of (4.7) above shows also for all $\mathcal{Y} \subset \mathcal{X}_{n}$ that

$$
\operatorname{Vol}_{n, 2}(\mathcal{Y}) \leq\left(1+\tilde{\gamma}_{n}\right)\left(1+\gamma_{n}\right) n \sum_{X \in \mathcal{Y}} r_{n}^{d} h_{n}(X)
$$

Since $h_{n}$ is bounded uniformly in $x$ and $n$, this implies the second inequality of (4.3). The first inequality of (4.3) is obtained similarly from (4.5): note that $h_{n}$ can be shown to be uniformly bounded away from zero, using the assumptions that $\rho_{\min }>0$ and $D$ has Lipschitz boundary. 
By adapting the proof of Lemma 4.2, we can obtain the following, which will be used in Section 5. The sequence $\left(\gamma_{n}\right)_{n \geq 1}$ is chosen as described at the start of Section 3. We shall need the factor of $\left(1-2 \gamma_{n}\right)$ (rather than just $\left.1-\gamma_{n}\right)$ in (4.9) when we use this result later on.

Lemma 4.3. Almost surely, the following holds. For any infinite $\mathcal{N} \subset \mathbb{N}$ and any sequence $\left(I_{n}\right)_{n \in \mathcal{N}}$ of subsets of $S_{n}$ such that the set $B_{n}:=\cup_{i \in I_{n}} Q_{i, n}$ satisfies $\inf _{n \in \mathcal{N}} \nu\left(B_{n}\right)>0$, and any sequence $\left(\mathcal{U}_{n}\right)_{n \in \mathcal{N}}$ of subsets of $\mathcal{X}_{n} \cap B_{n}$ with

$$
\left(1-2 \gamma_{n}\right) n \nu\left(Q_{i, n}\right) \leq\left|\mathcal{U}_{n} \cap Q_{i, n}\right| \leq\left(1+\gamma_{n}\right) n \nu\left(Q_{i, n}\right), \quad \forall i \in I_{n}, n \in \mathcal{N},
$$

it is the case that

$$
\lim _{n \rightarrow \infty, n \in \mathcal{N}}\left(\frac{\operatorname{Vol}_{n, v}\left(\mathcal{U}_{n}\right) \operatorname{Vol}_{\nu, v}(D)}{\operatorname{Vol}_{n, v}\left(\mathcal{X}_{n}\right) \operatorname{Vol}_{\nu, v}\left(B_{n}\right)}\right)=1, \quad v=1,2 .
$$

Proof. The result is trivial for $v=1$, so it suffices to consider the case with $v=2$. Given $\varepsilon>0$, let $D_{\varepsilon}$ denote the set of points $x \in D$ lying at Euclidean distance at least $\varepsilon$ from $\mathbb{R}^{d} \backslash D$. Then $D_{\varepsilon / 2}$ is compact so $\rho$ is uniformly continuous on $D_{\varepsilon / 2}$. Set

$$
B_{n, \varepsilon}:=\cup\left\{Q_{i, n}: i \in I_{n}, Q_{i, n} \cap D_{\varepsilon} \neq \emptyset\right\},
$$

which is contained in $D_{\varepsilon / 2}$ for large enough $n$.

In the proof of Lemma 4.2, note that if $X \in \mathcal{X}_{n}$ and $Q_{i, n}$ is the box containing $X$, then for any $z \in Q_{i, n}$ the inequalities (4.4) hold with the $X$ on the right replaced by $z$ (both times) because the function $\phi^{(n)}$ is constant on products of boxes. Therefore setting

$$
\underline{h}_{n}(x):=\inf _{z \in Q_{i, n}} h_{n}(z), \quad x \in Q_{i, n},
$$

we have as in (4.7) that

$$
\begin{aligned}
\operatorname{Vol}_{n, 2}\left(\mathcal{U}_{n} \cap B_{n, \varepsilon}\right) & \leq\left(1+\tilde{\gamma}_{n}\right)\left(1+\gamma_{n}\right) n r_{n}^{d} \sum_{X \in \mathcal{U}_{n} \cap B_{n, \varepsilon}} \underline{h}_{n}(X) \\
& \leq\left(1+\tilde{\gamma}_{n}\right)\left(1+\gamma_{n}\right)^{2} n^{2} r_{n}^{d} \int_{B_{n, \varepsilon}} \underline{h}_{n}(x) \nu(d x),
\end{aligned}
$$

where the last line comes from (4.9).

As discussed in the proof of Lemma 4.2, we have $h_{n}(x) \rightarrow h(x):=\rho(x) I(\phi)$ for all $x \in D$, where $I(\phi):=\int \phi(\|z\|) d z$. Moreover, we have the uniform convergence $\sup _{x \in B_{n, \varepsilon}}\left|h(x)-\underline{h}_{n}(x)\right| \rightarrow 0$. This can be seen from (4.6), using the fact that $\rho$ is uniformly continuous on $D_{\varepsilon / 2}$ (most easily by first considering the case where $\phi$ has bounded support). We therefore have that

$$
\operatorname{Vol}_{n, 2}\left(\mathcal{U}_{n} \cap B_{n, \varepsilon}\right) \leq(1+o(1)) n^{2} r_{n}^{d} \int_{B_{n, \varepsilon}} h(x) \nu(d x)
$$


Since $\nu\left(D \backslash D_{\varepsilon}\right) \rightarrow 0$ as $\varepsilon \downarrow 0$, given $\delta>0$, using (4.3) we may choose $\varepsilon>0$ so that

$$
\limsup _{n \rightarrow \infty}\left(n^{-2} r_{n}^{-d} \operatorname{Vol}_{n, 2}\left(\mathcal{U}_{n} \backslash B_{n, \varepsilon}\right)\right)<\delta .
$$

Combined with (4.11) and using the assumption that $\nu\left(B_{n}\right)$ is bounded away from zero for $n \in \mathbb{N}$, this shows that

$$
\limsup _{n \rightarrow \infty, n \in \mathbb{N}}\left(\frac{\operatorname{Vol}_{n, 2}\left(\mathcal{U}_{n}\right)}{n^{2} r_{n}^{d} \int_{B_{n}} h(x) \nu(d x)}\right) \leq 1 .
$$

By a similar argument one may show an inequality the other way for the limit inferior, and therefore the fraction in the left hand side of (4.12) actually tends to 1 as $n \rightarrow \infty$ through $\mathcal{N}$. Hence by (2.11),

$$
\frac{\operatorname{Vol}_{n, 2}\left(\mathcal{U}_{n}\right) \operatorname{Vol}_{\nu, 2}(D)}{\operatorname{Vol}_{n, 2}\left(\mathcal{X}_{n}\right) \operatorname{Vol}_{\nu, 2}\left(B_{n}\right)} \sim \frac{\operatorname{Vol}_{n, 2}\left(\mathcal{U}_{n}\right)}{n^{2} r_{n}^{d} I(\phi) \int_{B_{n}} \rho(x)^{2} d x} \rightarrow 1
$$

as $n \rightarrow \infty$ through $\mathcal{N}$. Thus we have the case $v=2$ of (4.10).

Lemma 4.4. Let $A \in \mathcal{B}(D)$ with $0<\nu(A)<1$. Let $(v, b) \in\{1,2\}^{2}$. For $n \in \mathbb{N}$ set $\mathcal{Y}_{n}:=\mathcal{X}_{n} \cap A$. Then as $n \rightarrow \infty$,

$$
\left(n^{2} r_{n}^{d+1}\right)^{-1}\left(\frac{\operatorname{Cut}_{n, \phi}\left(\mathcal{Y}_{n}\right)}{\operatorname{Bal}_{n, v, b}\left(\mathcal{Y}_{n}\right)}\right) \rightarrow \frac{\left(\sigma_{\phi} / 2\right) T V\left(\mathbf{1}_{A}\right)}{\operatorname{Bal}_{\nu, v, b}(A)}, \quad \text { a.s. }
$$

Proof. By the strong law of large numbers (for $v=1$ ) or by Lemma 4.2 (for $v=2$ ),

$$
\lim _{n \rightarrow \infty} \operatorname{Bal}_{n, v, b}\left(\mathcal{Y}_{n}\right)=\operatorname{Bal}_{\nu, v, b}(A), \quad(v, b) \in\{1,2\}^{2} .
$$

Therefore it suffices to show that

$$
\left(n^{2} r_{n}^{d+1}\right)^{-1} \operatorname{Cut}_{n, \phi}\left(\mathcal{Y}_{n}\right) \rightarrow\left(\sigma_{\phi} / 2\right) T V\left(\mathbf{1}_{A}\right), \quad \text { a.s. }
$$

The convergence of expectations corresponding to (4.13) follows from taking $u=\mathbf{1}_{A}$ in Part (ii) of Lemma 3.3.

The almost sure convergence in (4.13) was proved in [18] for the case where $\rho$ is constant on $D$ and $\phi=\phi_{U}$. In Remark 1.10 of [18] it is stated that the proof carries through to more general $\rho$ and to all weight functions $\phi$ satisfying (2.6)-(2.8). A similar result, with a more restricted range of sequences $\left(r_{n}\right)$ than we consider here, is given in Theorem 1 of [1].

The result (4.13) can alternatively be proved using a similar argument to the proof of Theorem 3.17 of [24], at least when $\phi$ has bounded support.

Proof of Proposition 4.1. Immediate from Lemma 4.4. 


\section{Lower bound}

In this section we complete the proof of Theorem 2.1. We shall also prove Theorem 2.2. Let $D, \rho, \phi$ and $\left(r_{n}\right)_{n \geq 1}$ be given, satisfying the assumptions of Theorem 2.1. Let $(v, b) \in\{1,2\}^{2}$. If $\mathrm{CHE}_{v, b}(D, \rho)=0$ then (2.10) is immediate from Proposition 4.1, so we assume until the end of the proof of Theorem 2.1 that $\mathrm{CHE}_{v, b}(D, \rho)>0$.

Our argument is related to one seen in in [13. Given $\mathcal{Y} \subset \mathcal{X}_{n}$, think of points in $\mathcal{Y}$ as being black and points of $\mathcal{X}_{n} \backslash \mathcal{Y}$ as being white. For $n \in \mathbb{N}$ and $i \in S_{n}$ (defined in Section [3), we shall say that the box $Q_{i, n}$ is grey (with respect to $\mathcal{Y}$ ) if both the number of black points in $Q_{i, n}$, and the number of white points in $Q_{i, n}$, exceed $\gamma_{n} n \nu\left(Q_{i, n}\right)$. We shall say the box $Q_{i, n}$ is black (with respect to $\mathcal{Y}$ ) if it is not grey and $\left|\mathcal{Y} \cap Q_{i, n}\right| \geq\left(1-2 \gamma_{n}\right) n \nu\left(Q_{i, n}\right)$. We shall say $Q_{i, n}$ is white (with respect to $\mathcal{Y})$ if it is not grey and $\left|\mathcal{X}_{n} \backslash \mathcal{Y}\right| \cap Q_{i, n} \geq\left(1-2 \gamma_{n}\right) n \nu\left(Q_{i, n}\right)$. By (3.13), for $n \geq N$ every box is either black, white or grey.

Let $g_{n}(\mathcal{Y})$ denote the number of grey boxes with respect to $\mathcal{Y}$. In other words, set

$$
g_{n}(\mathcal{Y}):=\sum_{i \in S_{n}} 1\left\{\min \left(\left|\mathcal{Y} \cap Q_{i, n}\right|,\left|\left(\mathcal{X}_{n} \backslash \mathcal{Y}\right) \cap Q_{i, n}\right|\right)>\gamma_{n} n \nu\left(Q_{i, n}\right)\right\}
$$

Define the within-box edges of $G_{\phi}\left(\mathcal{X}_{n}, r_{n}\right)$ to be those edges $\{x, y\}$ such that $\{x, y\} \subset Q_{i, n}$ for some $i$ (i.e., such that both endpoints lie in the same box), and let all other edges of $G_{\phi}\left(\mathcal{X}_{n}, r_{n}\right)$ be called between-box edges.

By (3.3) there exists $n_{2} \in \mathbb{N}$ such that for $n \geq n_{2}$, every within-box edge has weight at least $\phi(0) / 2$ in $G_{\phi}\left(\mathcal{X}_{n}, r_{n}\right)$, that is

$$
\phi\left(\frac{\|x-y\|}{r_{n}}\right) \geq \frac{\phi(0)}{2}, \quad \forall i \in S_{n}, x, y \in Q_{i, n}, n \geq n_{2}
$$

Let $\left(\mathcal{Y}_{n}\right)_{n \in \mathbb{N}}$ be a sequence of non-empty proper subsets of $\mathcal{X}_{n}$, each of which satisfies $\operatorname{Vol}_{n, v}\left(\mathcal{Y}_{n}\right) \leq \operatorname{Vol}_{n, v}\left(\mathcal{X}_{n}\right) / 2$ and achieves the minimum in (2.3) , i.e.

$$
\frac{\operatorname{Cut}_{n, \phi}\left(\mathcal{Y}_{n}\right)}{\operatorname{Bal}_{n, v, b}\left(\mathcal{Y}_{n}\right)}=\operatorname{CHE}_{v, b}\left(G_{\phi}\left(\mathcal{X}_{n}, r_{n}\right)\right)
$$

Lemma 5.1. Almost surely, it is the case that

$$
\limsup _{n \rightarrow \infty}\left(\frac{\mathrm{Cut}_{n, \phi}\left(\mathcal{Y}_{n}\right)}{n^{2} r_{n}^{d+1} \operatorname{Bal}_{n, v, b}\left(\mathcal{Y}_{n}\right)}\right) \leq\left(\sigma_{\phi} / 2\right) \operatorname{CHE}_{v, b}(D, \rho),
$$

and that there exists $n_{3} \in \mathbb{N}$ such that for all $n \in \mathbb{N}$ with $n \geq n_{3}$, at least one box is black and at least one box is white with respect to $\mathcal{Y}_{n}$. 
Proof. The first statement (5.3) follows from (5.2) and (4.1).

First suppose $v=1$. Suppose for infinitely many $n$ that there is no black box with respect to $\mathcal{Y}_{n}$. Then every box is grey or white, so each vertex in $\mathcal{Y}_{n}$ has at least $\gamma_{n} \rho_{\min } n\left(\gamma_{n} r_{n}\right)^{d}$ within-box white neighbours, and therefore by (5.1),

$$
\frac{\left(n^{2} r_{n}^{d+1}\right)^{-1} \operatorname{Cut}_{n, \phi}\left(\mathcal{Y}_{n}\right)}{\left(\left|\mathcal{Y}_{n}\right| / n\right)} \geq \gamma_{n}^{d+1} \rho_{\min } r_{n}^{-1} \phi(0) / 2,
$$

and by (3.1) this contradicts (5.3), whether we take $b=1$ or $b=2$, since the right hand side of (5.3) is finite. Similarly, for large enough $n \in \mathbb{N}$ at least one box is white.

Now suppose instead that $v=2$. By (4.3) and (2.11),

$$
\frac{\operatorname{Vol}_{n, 2}\left(\mathcal{Y}_{n}\right)}{\operatorname{Vol}_{n, 2}\left(\mathcal{X}_{n}\right)}=O\left(\frac{\left|\mathcal{Y}_{n}\right|}{n}\right)
$$

so we can argue similarly to the case already considered.

We now define a modification $\overline{\mathrm{Vol}}_{n, v}$ of the set function $\mathrm{Vol}_{n, v}$ with better linearity properties. For $x, y \in D$, recalling the definition (3.4) of the function $\phi_{n}(x, y)$, define

$$
\tilde{\phi}_{n}(x, y)= \begin{cases}0 & \text { if } x, y \text { lie in the same box } \\ \phi_{n}(x, y) & \text { otherwise. }\end{cases}
$$

For $\mathcal{Y} \subset \mathcal{X}_{n}$, define

$$
\overline{\operatorname{Vol}}_{n, v}(\mathcal{Y}):= \begin{cases}\sum_{y \in \mathcal{Y}} \sum_{x \in \mathcal{X}_{n} \backslash\{y\}} \tilde{\phi}_{n}(x, y) & \text { if } v=2 \\ \operatorname{Vol}_{n, 1}(\mathcal{Y}) & \text { if } v=1 .\end{cases}
$$

Lemma 5.2. There exist constants $\delta_{n} \downarrow 0$ such that almost surely, for all $n$ and all $\mathcal{Y} \subset \mathcal{X}_{n}$

$$
\operatorname{Vol}_{n, v}(\mathcal{Y}) \leq\left(1+\delta_{n}\right) \overline{\operatorname{Vol}}_{n, v}(\mathcal{Y}), \quad v=1,2
$$

Proof. It suffices to consider the case $v=2$. For $x \in D, \alpha \in(0, \pi)$ and $e \in \mathbb{R}^{d}$ with $\|e\|=1$, let $\mathcal{K}(x, e, \alpha)$ denote the open cone consisting of those $y \in \mathbb{R}^{d} \backslash\{x\}$ such that the vector $y-x$ makes an angle less than $\alpha$ with $e$. For $r>0$ let $B(x ; r):=\left\{y \in \mathbb{R}^{d}:\|y-x\| \leq r\right\}$, and let $\omega_{d}$ denote the Lebesgue measure of $B(x ; 1)$.

By the assumption that $D$ has Lipschitz boundary, and a compactness argument, we can (and do) choose $\alpha \in(0,1 / 6)$ and $r_{0}>0$ such that for all $x \in D$ there exists $e(x) \in \mathbb{R}^{d}$ with $\|e(x)\|=1$ such that $\mathcal{K}(x, e(x), 3 \alpha) \cap B\left(x ; r_{0}\right) \subset D$. 
Choose $a \in(0,1 / 4)$ with $\phi(2 a)>0$. For $x \in D$, and $n \in \mathbb{N}$ large enough so that $a r_{n}<r_{0} / 2$, note that $B\left(x+a r_{n} e(x) ; 3 a \alpha r_{n}\right) \subset D$. Then for large enough $n$ and all $x \in D$, for every $i \in S_{n}$ such that $Q_{i, n} \cap B\left(x+a r_{n} e(x) ; a \alpha r_{n}\right) \neq \emptyset$, we have that $Q_{i, n} \subset D$ and moreover $Q_{i, n}=Q_{i, n}^{\prime}$ (that is, $Q_{i, n}$ does not touch the boundary of $D)$, and furthermore for all $y \in Q_{i, n}$ we have $(a / 2) r_{n} \leq\|y-x\| \leq 2 a r_{n}$ so that $\phi_{n}(x, y) \geq \phi(2 a)$. Therefore summing over all such $i$ and using (3.13), we obtain for all $x \in \mathcal{X}_{n}$ that

$$
\overline{\operatorname{Vol}}_{n, 2}(\{x\}) \geq\left(1-\gamma_{n}\right) n \rho_{\min } \phi(2 a) \omega_{d}\left(a \alpha r_{n}\right)^{d},
$$

while using (3.3) and (3.12) we have that

$$
\operatorname{Vol}_{n, 2}(\{x\})-\overline{\operatorname{Vol}}_{n, 2}(\{x\}) \leq 2 n \rho_{\max }\left(2 C \gamma_{n} r_{n}\right)^{d} .
$$

Summing over $x \in \mathcal{Y}$ we obtain that

$$
\frac{\operatorname{Vol}_{n, 2}(\{\mathcal{Y}\})-\overline{\operatorname{Vol}}_{n, 2}(\{\mathcal{Y}\})}{\overline{\operatorname{Vol}}_{n, 2}(\{\mathcal{Y}\})} \leq \frac{2^{1+d} \rho_{\max } C^{d} \gamma_{n}^{d}}{\left(1-\gamma_{n}\right) \rho_{\min } \phi(2 a) \omega_{d}(\alpha a)^{d}}
$$

which tends to zero, as required.

For $n \in \mathbb{N}$ and $\mathcal{Y} \subset \mathcal{X}_{n}$, define the modified cut function

$$
\operatorname{Cut}_{n, \phi}^{\prime}(\mathcal{Y}):=\sum_{x \in \mathcal{Y}} \sum_{y \in \mathcal{X}_{n} \backslash \mathcal{Y}} \phi_{n}(x, y)
$$

Denote by $\mathcal{N}^{*}$ the set of $n \in \mathbb{N}$ such that $\operatorname{Vol}_{n, v}\left(\mathcal{Y}_{n}\right) / \operatorname{Vol}_{n, v}\left(\mathcal{X}_{n}\right) \in\left[\gamma_{n}, 1 / 2\right]$. The next lemma is a key part of our proof. It provides a method of 'greyscale removal' whereby we modify $\mathcal{Y}$ slightly in a manner that makes all the boxes black or white.

Lemma 5.3. Almost surely, there exists a sequence of subsets $\mathcal{Y}_{n}^{\prime} \subset \mathcal{X}_{n}$, defined for $n \geq 1$, satisfying

$$
n^{-1}\left|\mathcal{Y}_{n}^{\prime} \triangle \mathcal{Y}_{n}\right| \ll \gamma_{n}^{2}
$$

and a (random) number $n_{4} \in \mathbb{N}$ such that for all $n \in \mathbb{N}$ with $n \geq n_{4}$ we have that

$$
g_{n}\left(\mathcal{Y}_{n}^{\prime}\right)=0
$$

and that the union $B_{n}$ of black boxes induced by $\mathcal{Y}_{n}^{\prime}$ satisfies $B_{n} \neq \emptyset$ and $D \backslash B_{n} \neq \emptyset$, and moreover that

$$
\operatorname{Cut}_{n, \phi}\left(\mathcal{Y}_{n}\right) \geq \operatorname{Cut}_{n, \phi}^{\prime}\left(\mathcal{Y}_{n}^{\prime}\right) \quad \text { if } n \in \mathcal{N}^{*}
$$

and

$$
\frac{\operatorname{Cut}_{n, \phi}\left(\mathcal{Y}_{n}\right)}{\overline{\operatorname{Vol}}_{n, v}\left(\mathcal{Y}_{n}\right)} \geq \frac{Z_{n}}{\overline{\operatorname{Vol}}_{n, v}\left(\mathcal{Y}_{n}^{\prime} \cap B_{n}\right)} \quad \text { if } n \in \mathbb{N} \backslash \mathcal{N}^{*},
$$

where $Z_{n}$ denotes the contribution to $\mathrm{Cut}_{n, \phi}^{\prime}\left(\mathcal{Y}_{n}^{\prime}\right)$ from edges with exactly one endpoint in $B_{n}$. 
Proof. Set $K_{n}:=5 \rho_{\min }^{-2} \gamma_{n}^{-2 d-2} \sigma_{\phi} \operatorname{CHE}_{v, b}(D, \rho) / \phi(0)$, which tends to infinity since $\gamma_{n}$ tends to zero. Suppose there is an infinite set $\mathcal{N}_{1} \subset \mathbb{N}$ such that $g_{n}\left(\mathcal{Y}_{n}\right) \geq K_{n} r_{n}^{1-d}$ for all $n \in \mathcal{N}_{1}$. Then by considering only the within-box edges and using (5.1), we have for large enough $n \in \mathcal{N}_{1}$ that

$$
\begin{array}{r}
\operatorname{Cut}_{n, \phi}\left(\mathcal{Y}_{n}\right) \geq g_{n}\left(\mathcal{Y}_{n}\right)\left(\gamma_{n}^{d+1} \rho_{\min } n r_{n}^{d}\right)^{2} \phi(0) / 5 \\
\geq K_{n} \gamma_{n}^{2 d+2} \rho_{\min }^{2} n^{2} r_{n}^{d+1} \phi(0) / 5 \\
=n^{2} r_{n}^{d+1} \sigma_{\phi} \operatorname{CHE}_{v, b}(D, \rho),
\end{array}
$$

which would contradict (5.3) since $\operatorname{Bal}_{n, v, b}\left(\mathcal{Y}_{n}\right) \leq 1$. Therefore there exists $n_{5} \in$ $\left[n_{0}, \infty\right)$ such that

$$
g_{n}\left(\mathcal{Y}_{n}\right)<K_{n} r_{n}^{1-d} \text { for all } n \geq n_{5} .
$$

Suppose $\mathcal{N}^{*}$ is infinite and let $n \in \mathcal{N}^{*}\left(\operatorname{so~that~}^{\operatorname{Vol}_{n, v}}\left(\mathcal{Y}_{n}\right) / \operatorname{Vol}_{n, v}\left(\mathcal{X}_{n}\right) \in\left[\gamma_{n}, 1 / 2\right]\right)$. Assume also that $n \geq \max \left(N, n_{5}\right)$, where $N$ is as in Lemma 3.2. We consider the effect of changing the colour of some of the vertices in a given box, on the contribution of between-box edges to the cut. Let $i \in \mathbb{N}$. Suppose there are $\ell$ black vertices (with respect to $\mathcal{Y}_{n}$ ) and $w$ white vertices in the box $Q_{i, n}$, and recalling the definition of $z_{i, n}$ from the start of Section 3, set

$$
\begin{array}{r}
\ell^{\prime}:=\sum_{j \in S_{n} \backslash\{i\}} \phi_{n}\left(z_{i, n}, z_{j, n}\right)\left|\mathcal{Y}_{n} \cap Q_{j, n}\right| ; \\
w^{\prime}:=\sum_{j \in S_{n} \backslash\{i\}} \phi_{n}\left(z_{i, n}, z_{j, n}\right)\left|\left(\mathcal{X}_{n} \backslash \mathcal{Y}_{n}\right) \cap Q_{j, n}\right| .
\end{array}
$$

Let $m:=\ell+w$, the total number of vertices in $Q_{i, n}$. Then the total contribution to $\operatorname{Cut}_{n, \phi}^{\prime}\left(\mathcal{Y}_{n}\right)$ from between-box edges with one endpoint in $Q_{i, n}$ is equal to the expression

$$
\ell w^{\prime}+w \ell^{\prime}=\ell w^{\prime}+(m-\ell) \ell^{\prime}=\ell\left(w^{\prime}-\ell^{\prime}\right)+m \ell^{\prime} .
$$

This expression is a linear function of $\ell$, if we consider $m, w^{\prime}$ and $\ell^{\prime}$ as being fixed. Therefore as a function of $\ell$ it is minimised over the range $[0, m]$ either at $\ell=0$ or at $\ell=m$ (or both). Moreover, taking $\ell=0$ or $\ell=m$ reduces the number of within-box edges in this box to zero. Hence we can (and do) modify the colour of vertices in $Q_{i, n}$ to make all vertices in $Q_{i, n}$ have the same colour, in such a way that resulting set $\tilde{\mathcal{Y}}_{n}$ of black vertices has $\operatorname{Cut}_{n, \phi}^{\prime}\left(\tilde{\mathcal{Y}}_{n}\right) \leq \operatorname{Cut}_{n, \phi}^{\prime}\left(\mathcal{Y}_{n}\right)$.

Repeating this process for each of the $i$ such that $Q_{i, n}$ is grey with respect to the original set $\mathcal{Y}_{n}$, considered one by one, we end up with a new set of black vertices, denoted $\mathcal{Y}_{n}^{\prime}$, with $g_{n}\left(\mathcal{Y}_{n}^{\prime}\right)=0$, such that $\operatorname{Cut}_{n, \phi}^{\prime}\left(\mathcal{Y}_{n}^{\prime}\right) \leq \operatorname{Cut}_{n, \phi}^{\prime}\left(\mathcal{Y}_{n}\right)$. Also $\operatorname{Cut}_{n, \phi}^{\prime}\left(\mathcal{Y}_{n}\right) \leq \operatorname{Cut}_{n, \phi}\left(\mathcal{Y}_{n}\right)$ by (5.6) and (3.6)), so we have (5.9). 
Next we prove (5.10). Suppose $\mathbb{N} \backslash \mathcal{N}^{*}$ is infinite (else (5.10) holds vacuously for large enough $n$ ). Let $n \in \mathbb{N} \backslash \mathcal{N}^{*}$. Assume $n \geq n_{3}$ with $n_{3}$ given by Lemma 5.1, so there is at least one black box and at least one white box with respect to $\mathcal{Y}_{n}$. Let us write $x_{n, 1}$ for the total weight (using weight function $\phi_{n}$ ) of between-box cut edges involving black vertices in black boxes, $x_{n, 2}$ for the total weight of between-box cut edges involving black vertices in grey boxes and $x_{n, 3}$ for the total weight of withinbox cut edges involving black vertices in white boxes. Let $y_{n, 1}, y_{n, 2}, y_{n, 3}$ be the total volume $\overline{\mathrm{Vol}}_{n, v}$ (as defined in (5.4) of the set of black vertices in black boxes, in grey boxes and in white boxes respectively. Set $V_{n}:=\operatorname{Vol}_{n, v}\left(\mathcal{X}_{n}\right)$.

Then

$$
\frac{\mathrm{Cut}_{n, \phi}^{\prime}\left(\mathcal{Y}_{n}\right)}{\overline{\operatorname{Vol}}_{n, v}\left(\mathcal{Y}_{n}\right) / V_{n}} \geq \frac{V_{n} x_{n, 1}+V_{n} x_{n, 2}+V_{n} x_{n, 3}}{y_{n, 1}+y_{n, 2}+y_{n, 3}} \geq \min \left(\frac{V_{n} x_{n, 1}+V_{n} x_{n, 2}}{y_{n, 1}+y_{n, 2}}, \frac{V_{n} x_{n, 3}}{y_{n, 3}}\right) .
$$

By a similar argument to the proof of Lemma 5.1 (see also Lemma 4.2), we have that $\left(n^{2} r_{n}^{d+1}\right)^{-1}\left(V_{n} x_{n, 3} / y_{n, 3}\right)$ exceeds a strictly positive constant times $\gamma_{n}^{d+1} r_{n}^{-1}$, and therefore by (5.3), for large enough $n \in \mathbb{N} \backslash \mathcal{N}^{*}$ the above minimum must be achieved by the the first of the two ratios.

We now look again at the effect of changing the the colour of vertices in a grey box $Q_{i, n}$. With $w^{\prime}$ defined by (5.13), let $\ell^{\prime \prime}$ be defined similarly to $\ell^{\prime}$ in (5.12) but with the sum restricted to those $j \in S_{n} \backslash\{i\}$ for which the box $Q_{j, n}$ is black or grey. Then set $\alpha:=w^{\prime}-\ell^{\prime \prime}$. Write $x$ for the expression denoted $x_{n, 1}+x_{n, 2}$ above and $y$ for $y_{n, 1}+y_{n, 2}$. If we change the number of black vertices in the box by amount $k$, keeping the total number of vertices the same (in fact we shall consider just two possible values of $k$ below), then the value of $x$ changes to $x+\alpha k:=x^{\prime}$ and $y$ changes to $y+\beta k:=y^{\prime}$, where we set

$$
\beta= \begin{cases}1 & \text { if } v=1 \\ \sum_{j \in S_{n}} \tilde{\phi}_{n}\left(z_{i, n}, z_{j, n}\right)\left|\mathcal{Y}_{n} \cap Q_{j, n}\right| & \text { if } v=2 .\end{cases}
$$

Then

$$
\frac{x^{\prime}}{y^{\prime}}-\frac{x}{y}=\frac{x+\alpha k}{y+\beta k}-\frac{x}{y}=\frac{(x+\alpha k) y-x(y+\beta k)}{(y+\beta k) y}=\frac{k(\alpha y-\beta x)}{(y+\beta k) y},
$$

which can be made non-positive either by taking $k=\left|\left(\mathcal{X}_{n} \backslash \mathcal{Y}_{n}\right) \cap Q_{i, n}\right|$ or by taking $k=-\left|\mathcal{Y}_{n} \cap Q_{i, n}\right|$ (depending on the sign of $\alpha y-\beta x$ ). Note that since there is at least one black box and every box has at least one neighbouring box, we have $y+\beta k>0$ for both of these choices of $k$.

Therefore we can choose a colour (white or black) and change all the vertices in $Q_{i, n}$ to that colour, without increasing the ratio $x / y$. Repeating this for each of the grey boxes in turn, we end up with a set $\mathcal{Y}_{n}^{\prime}$ that induces no grey boxes and has a reduced (or at least not increased) value of $x / y$ compared to $\mathcal{Y}_{n}$. Also, this 
procedure does not affect the value of $x_{n, 3}$ or $y_{n, 3}$ because, while some new white boxes might be created, none of them contains black vertices at all. Let $y_{n, 1}^{\prime}$ be the number of black vertices in black boxes induced by $\mathcal{Y}_{n}^{\prime}$, and let $x_{n, 1}^{\prime}$ be the total $\phi_{n}$-weight of between-box cut edges involving these vertices. Then

$$
\frac{\operatorname{Cut}_{n, \phi}\left(\mathcal{Y}_{n}\right)}{\overline{\operatorname{Vol}}_{n, v}\left(\mathcal{Y}_{n}\right) / V_{n}} \geq \frac{V_{n} x_{n, 1}+V_{n} x_{n, 2}}{y_{n, 1}+y_{n, 2}} \geq \frac{V_{n} x_{n, 1}^{\prime}}{y_{n, 1}^{\prime}},
$$

and (5.10) follows because $x_{n, 1}^{\prime} \geq Z_{n}$.

Finally, in both cases considered above $\left(n \in \mathcal{N}^{*}\right.$ and $\left.n \in \mathbb{N} \backslash \mathcal{N}^{*}\right)$, the modification of $\mathcal{Y}_{n}$ to obtain $\mathcal{Y}_{n}^{\prime}$ involves changing the colour only of vertices in grey boxes (with respect to $\mathcal{Y}_{n}$ ), so every black (repectively white) box with respect to $\mathcal{Y}_{n}$ is also black (resp. white) with respect to $\mathcal{Y}_{n}^{\prime}$. It then follows from Lemma 5.1 that almost surely, $B_{n}$ and $D \backslash B_{n}$ are non-empty for large enough $n$. Moreover, for large enough $n$, by (5.11) and (3.12),

$$
\begin{aligned}
n^{-1}\left|\mathcal{Y}_{n}^{\prime} \triangle \mathcal{Y}_{n}\right| & \leq 2 K_{n} \gamma_{n}^{d} \rho_{\max }(2 C)^{d} r_{n} \\
& =O\left(\gamma_{n}^{-d-2} r_{n}\right) .
\end{aligned}
$$

Thus by (3.1) we have (5.7).

As in the statement of Lemma 5.3, for $n \in \mathbb{N}$ let $B_{n}$ denote the union of the black boxes induced by $\mathcal{Y}_{n}^{\prime}$, let $Z_{n}$ be the contribution to $\operatorname{Cut}_{n, \phi}^{\prime}\left(\mathcal{Y}_{n}^{\prime}\right)$ from edges with one endpoint in $B_{n}$ and the other endpoint in $W_{n}:=D \backslash B_{n}$, the union of the white boxes. Then by (3.13),

$$
Z_{n} \geq\left(1-2 \gamma_{n}\right)^{2} n^{2}\left(\int_{B_{n}} \int_{W_{n}} \phi_{n}(x, y) \nu(d x) \nu(d y)\right) .
$$

Therefore by Lemma 3.1, with $C^{\prime}, \tilde{\gamma}_{n}$ and $\tilde{r}_{n}$ as defined in that result,

$$
\begin{array}{r}
Z_{n} \geq\left(1-2 \gamma_{n}\right)^{2}\left(1-\tilde{\gamma}_{n}\right) n^{2}\left(\int_{B_{n}} \int_{W_{n}} \phi\left(\frac{\|y-x\|}{\tilde{r}_{n}}\right) \nu(d x) \nu(d y)\right) \\
=\left(1-2 \gamma_{n}\right)^{2}\left(1-\tilde{\gamma}_{n}\right) n^{2} \tilde{r}_{n}^{d+1}\left((1 / 2) T V_{\phi, \tilde{r}_{n}}\left(\mathbf{1}_{B_{n}}\right)\right)
\end{array}
$$

where $T V_{\phi, r}(u)$ is as defined in (3.15).

Lemma 5.4. Almost surely $\mathbb{N} \backslash \mathcal{N}^{*}$ is finite.

Proof. Suppose $\mathbb{N} \backslash \mathcal{N}^{*}$ is infinite. Let $n \in \mathbb{N} \backslash \mathcal{N}^{*}$, so $\operatorname{Vol}_{n, v}\left(\mathcal{Y}_{n}\right)<\gamma_{n} \operatorname{Vol}_{n, v}\left(\mathcal{X}_{n}\right)$. Set $V_{n}:=\operatorname{Vol}_{n, v}\left(\mathcal{X}_{n}\right)$. By (2.1), (2.2) and (5.5),

$$
\operatorname{Bal}_{n, v, b}\left(\mathcal{Y}_{n}\right) \leq \operatorname{Vol}_{n, v}\left(\mathcal{Y}_{n}\right) / V_{n} \leq\left(1+\delta_{n}\right) \overline{\operatorname{Vol}}_{n, v}\left(\mathcal{Y}_{n}\right) / V_{n}
$$


Therefore using (5.2) and (5.10), we have for $b=1,2$ that

$$
\mathrm{CHE}_{v, b}\left(G_{\phi}\left(\mathcal{X}_{n}, r_{n}\right)\right) \geq \frac{\left(1+\delta_{n}\right)^{-1} \operatorname{Cut}_{n, \phi}\left(\mathcal{Y}_{n}\right)}{\overline{\operatorname{Vol}}_{n, v}\left(\mathcal{Y}_{n}\right) / V_{n}} \geq \frac{\left(1+\delta_{n}\right)^{-1} Z_{n}}{\overline{\operatorname{Vol}}_{n, v}\left(\mathcal{Y}_{n}^{\prime} \cap B_{n}\right) / V_{n}} .
$$

By (3.12), if $v=1$ then $\operatorname{Vol}_{n, 1}\left(\mathcal{Y}_{n}^{\prime} \cap B_{n}\right) \leq 2 n \nu\left(B_{n}\right)$ for $n$ large; if $v=2$ then using (4.3) and (2.11) we can find a constant $C^{\prime \prime \prime}$ such that for all large enough $n$ we have

$$
\operatorname{Vol}_{n, 2}\left(\mathcal{Y}_{n}^{\prime} \cap B_{n}\right) / V_{n} \leq \frac{C^{\prime \prime}\left|\mathcal{Y}_{n}^{\prime} \cap B_{n}\right|}{n \int \rho(x)^{2} d x \int \phi(\|y\|) d y} \leq C^{\prime \prime \prime}\left(1+\gamma_{n}\right) \nu\left(B_{n}\right) .
$$

Hence by (5.14) and (5.15), for every $(v, b) \in\{1,2\}^{2}$ there exists a constant $c>0$ such that for all large enough $n$,

$$
\frac{\mathrm{CHE}_{v, b}\left(G_{\phi}\left(\mathcal{X}_{n}, r_{n}\right)\right)}{n^{2} r_{n}^{d+1}} \geq \frac{c T V_{\phi, \tilde{r}_{n}}\left(\mathbf{1}_{B_{n}}\right)}{2 \nu\left(B_{n}\right)}
$$

Since the functions $\mathbf{1}_{B_{n}} / \nu\left(B_{n}\right)$ are $L^{1}(\nu)$-bounded, uniformly in $n$, this shows by (4.1) and compactness (part (iii) of Lemma 3.3) that the functions $\mathbf{1}_{B_{n}} / \nu\left(B_{n}\right)$ converge in $L^{1}(\nu)$ along a subsequence to a limiting function of the form $\mathbf{1}_{B} / \nu(B)$ with $B \in \mathcal{B}(D)$ and $0<\nu(B)<1$; see Lemma 6 of [19].

However, by (5.7) and (in the case $v=2$ ) (4.3) we have that $n^{-1}\left|\mathcal{Y}_{n}^{\prime}\right| \rightarrow 0$ as $n \rightarrow \infty$ through $\mathbb{N} \backslash \mathcal{N}^{*}$. Then by (3.13), as $n \rightarrow \infty$ through $\mathbb{N} \backslash \mathcal{N}^{*}$ we have

$$
\nu\left(B_{n}\right) \leq\left(1-\gamma_{n}\right)^{-1} n^{-1}\left|\mathcal{Y}_{n}^{\prime}\right| \rightarrow 0,
$$

which contradicts the conclusion above that $\mathbf{1}_{B_{n}} / \nu\left(B_{n}\right)$ converges in $L^{1}$ to a limit of the form $\mathbf{1}_{B}$, for some Borel $B \subset D$ with $0<\nu(B)<1$. Therefore $\mathbb{N} \backslash \mathcal{N}^{*}$ must be finite.

Lemma 5.5. Let $\mathcal{Y}_{n}^{\prime}$ and $B_{n}$ be as in Lemma 5.3. Set $\mathcal{Y}_{n, B}^{\prime}:=\mathcal{Y}_{n}^{\prime} \cap B_{n}$. Then

$$
\frac{T V_{\phi, \tilde{r}_{n}}\left(\mathbf{1}_{B_{n}}\right)}{\operatorname{Bal}_{n, v, b}\left(\mathcal{Y}_{n, B}^{\prime}\right)} \leq(2+o(1))\left(\frac{\mathrm{CHE}_{v, b}\left(G_{\phi}\left(\mathcal{X}_{n}, r_{n}\right)\right)}{n^{2} r_{n}^{d+1}}\right)
$$

and

$$
\limsup _{n \rightarrow \infty}\left(\frac{T V_{\phi, \tilde{r}_{n}}\left(\mathbf{1}_{B_{n}}\right)}{\mathrm{Bal}_{n, v, b}\left(\mathcal{Y}_{n, B}^{\prime}\right)}\right) \leq \sigma_{\phi} \operatorname{CHE}_{v, b}(D, \rho)<\infty
$$

Proof. Set $V_{n}:=\operatorname{Vol}_{n, v}\left(\mathcal{X}_{n}\right)$ as before. For all but finitely many $n \in \mathbb{N}$ we have by Lemma 3.2 that $n \geq N$, and by Lemma 5.4 that $n \in \mathcal{N}^{*}$ (so that $\operatorname{Vol}_{n, v}\left(\mathcal{Y}_{n}\right) / V_{n} \in$ $\left.\left[\gamma_{n}, 1 / 2\right]\right)$, and by Lemma 5.3 that $g_{n}\left(\mathcal{Y}_{n}^{\prime}\right)=0$ so that for each $i \in S_{n}$ the box $Q_{i, n}$ 
is either black or white but not both (with respect to $\mathcal{Y}_{n}^{\prime}$ ), and the sets $B_{n}$ and $W_{n}:=D \backslash B_{n}$ are non-empty. Consider such $n$.

We claim next that whichever value of $(v, b) \in\{1,2\}^{2}$ we are considering, we have for large enough $n$ that

$$
\operatorname{Bal}_{n, v, b}\left(\mathcal{Y}_{n}^{\prime}\right) / \operatorname{Bal}_{n, v, b}\left(\mathcal{Y}_{n}\right) \geq 1-\gamma_{n}
$$

If $v=1$ this follows from (5.7) and the fact that $n \in \mathcal{N}^{*}$. If $v=2$, to see (5.18) we use also the fact that by (5.7) and (4.3) we have

$$
\left|\operatorname{Vol}_{n, 2, b}\left(\mathcal{Y}_{n}^{\prime}\right)-\operatorname{Vol}_{n, 2, b}\left(\mathcal{Y}_{n}\right)\right| \ll n^{2} r_{n}^{d} \gamma_{n}^{2}
$$

while $\operatorname{Vol}_{n, 2}\left(\mathcal{Y}_{n}\right) \geq c \gamma_{n} n^{2} r_{n}^{d}$ for some constant $c>0$, by the fact that $n \in \mathcal{N}^{*}$, and (2.11).

Hence for large enough $n$, by (5.2) and (5.9) we have

$$
\mathrm{CHE}_{v, b}\left(G_{\phi}\left(\mathcal{X}_{n}, r_{n}\right)\right) \geq \frac{\left(1-\gamma_{n}\right) \operatorname{Cut}_{n, \phi}^{\prime}\left(\mathcal{Y}_{n}^{\prime}\right)}{\operatorname{Bal}_{n, v, b}\left(\mathcal{Y}_{n}^{\prime}\right)}
$$

Let $\mathcal{Y}_{n, B}^{\prime}:=\mathcal{Y}_{n}^{\prime} \cap B_{n}$ and $\mathcal{Y}_{n, W}^{\prime}:=\mathcal{Y}_{n}^{\prime} \cap W_{n}$. Let $\partial_{n, \phi}^{W}\left(\mathcal{Y}_{n}^{\prime}\right)$ be the total $\phi_{n}$-weight of within-box edges from $\mathcal{Y}_{n, W}^{\prime}$ to $\mathcal{X}_{n} \backslash \mathcal{Y}_{n}^{\prime}$. Then

$$
\frac{\operatorname{Cut}_{n, \phi}^{\prime}\left(\mathcal{Y}_{n}^{\prime}\right)}{\operatorname{Vol}_{n, v}\left(\mathcal{Y}_{n}^{\prime}\right)} \geq \frac{Z_{n}+\partial_{n, \phi}^{W}\left(\mathcal{Y}_{n}^{\prime}\right)}{\operatorname{Vol}_{n, v}\left(\mathcal{Y}_{n, B}^{\prime}\right)+\operatorname{Vol}_{n, v}\left(\mathcal{Y}_{n, W}^{\prime}\right)} \geq \min \left(\frac{Z_{n}}{\operatorname{Vol}_{n, v}\left(\mathcal{Y}_{n, B}^{\prime}\right)}, \frac{\partial_{n, \phi}^{W}\left(\mathcal{Y}_{n}^{\prime}\right)}{\operatorname{Vol}_{n, v}\left(\mathcal{Y}_{n, W}^{\prime}\right)}\right)
$$

Hence

$$
\frac{\left(n^{2} r_{n}^{d+1}\right)^{-1} \operatorname{Cut}_{n, \phi}^{\prime}\left(\mathcal{Y}_{n}^{\prime}\right)}{\operatorname{Bal}_{n, v, 1}\left(\mathcal{Y}_{n}^{\prime}\right)} \geq \min \left(\frac{\left(n^{2} r_{n}^{d+1}\right)^{-1} Z_{n}}{\operatorname{Vol}_{n, v}\left(\mathcal{Y}_{n, B}^{\prime}\right) / V_{n}}, \frac{\left(n^{2} r_{n}^{d+1}\right)^{-1} \partial_{n, \phi}^{W}\left(\mathcal{Y}_{n}^{\prime}\right)}{\operatorname{Vol}_{n, v}\left(\mathcal{Y}_{n, W}^{\prime}\right) / V_{n}}\right)
$$

Each vertex $x \in \mathcal{Y}_{n, W}^{\prime}$ has at least $\gamma_{n}^{d}\left(1-2 \gamma_{n}\right) \rho_{\min } n r_{n}^{d}$ within-box neighbours in $\mathcal{X}_{n} \backslash \mathcal{Y}_{n}^{\prime}$. Therefore there is a constant $c^{\prime}>0$ such that for $n$ large enough, the second ratio inside the minimum in the right hand side of (5.20) is at least $c^{\prime} \gamma_{n}^{d}(1-$ $\left.2 \gamma_{n}\right) \rho_{\min } r_{n}^{-1} \phi(0)$; we may take $c^{\prime}=1 / 2$ for $v=1$ and otherwise use the fact that by (4.3) and (2.11) we have

$$
\operatorname{Vol}_{n, 2}\left(\mathcal{Y}_{n, W}^{\prime}\right) / \operatorname{Vol}_{n, 2}\left(\mathcal{X}_{n}\right) \leq \text { const. } \times \operatorname{Vol}_{n, 1}\left(\mathcal{Y}_{n, W}^{\prime}\right) / \operatorname{Vol}_{n, 1}\left(\mathcal{X}_{n}\right)
$$

Hence this second ratio tends to infinity by (3.1). By (5.19) and (4.1), if $b=1$ then the left hand side of (5.20) is bounded, so for large enough $n$ the minimum is achieved by the first of the two ratios in the right hand side of (5.20). 
Also,

$$
\begin{array}{r}
\frac{\operatorname{Cut}_{n, \phi}^{\prime}\left(\mathcal{Y}_{n}^{\prime}\right)}{\left(\frac{\operatorname{Vol}_{n, v}\left(\mathcal{Y}_{n}^{\prime}\right)}{V_{n}}\right)\left(1-\frac{\operatorname{Vol}_{n, v}\left(\mathcal{Y}_{n}^{\prime}\right)}{V_{n}}\right)} \geq \frac{Z_{n}+\partial_{n}^{W}\left(\mathcal{Y}_{n}^{\prime}\right)}{\left(\frac{\operatorname{Vol}_{n, v}\left(\mathcal{Y}_{n, B}^{\prime}\right)}{V_{n}}+\frac{\operatorname{Vol}_{n, v}\left(\mathcal{Y}_{n, W}^{\prime}\right)}{V_{n}}\right)\left(1-\frac{\operatorname{Vol}_{n, v}\left(\mathcal{Y}_{n}^{\prime}\right)}{V_{n}}\right)} \\
\geq \min \left(\frac{Z_{n}}{\left(\frac{\operatorname{Vol}_{n, v}\left(\mathcal{Y}_{n, B}^{\prime}\right)}{V_{n}}\right)\left(1-\frac{\operatorname{Vol}_{n, v}\left(\mathcal{Y}_{n}^{\prime}\right)}{V_{n}}\right)}, \frac{\partial_{n}^{W}\left(\mathcal{Y}_{n}^{\prime}\right)}{\left.\frac{\operatorname{Vol}_{n, v}\left(\mathcal{Y}_{n, W}^{\prime}\right)}{V_{n}}\right)}\right),
\end{array}
$$

and similarly to before, if $b=2$ then for large enough $n$ the minimum is achieved by the first term. Thus using (5.20) for $b=1$ and (5.21) for $b=2$, in both cases we have for large enough $n$ that

$$
\frac{\operatorname{Cut}_{n, \phi}^{\prime}\left(\mathcal{Y}_{n}^{\prime}\right)}{\operatorname{Bal}_{n, v, b}\left(\mathcal{Y}_{n}^{\prime}\right)} \geq \frac{Z_{n}}{\operatorname{Bal}_{n, v, b}\left(\mathcal{Y}_{n, B}^{\prime}\right)} \text {. }
$$

Therefore using (5.14) followed by (5.19) we have that

$$
\begin{array}{r}
\frac{T V_{\phi, \tilde{r}_{n}}\left(\mathbf{1}_{B_{n}}\right)}{\mathrm{Bal}_{n, v, b}\left(\mathcal{Y}_{n, B}^{\prime}\right)} \leq \frac{T V_{\phi, \tilde{r}_{n}}\left(\mathbf{1}_{B_{n}}\right) \mathrm{Cut}_{n, \phi}^{\prime}\left(\mathcal{Y}_{n}^{\prime}\right)}{Z_{n} \operatorname{Bal}_{n, v, b}\left(\mathcal{Y}_{n}^{\prime}\right)} \leq \frac{(2+o(1))\left(n^{2} r_{n}^{d+1}\right)^{-1} \mathrm{Cut}_{n, \phi}^{\prime}\left(\mathcal{Y}_{n}^{\prime}\right)}{\operatorname{Bal}_{n, v, b}\left(\mathcal{Y}_{n}^{\prime}\right)} \\
\leq(2+o(1))\left(n^{2} r_{n}^{d+1}\right)^{-1} \mathrm{CHE}_{v, b}\left(G_{\phi}\left(\mathcal{X}_{n}, r_{n}\right)\right) .
\end{array}
$$

This gives us (5.16), and then (5.17) follows from Proposition 4.1.

Using Lemma 4.3 we obtain the following.

Lemma 5.6. Let $B_{n}$ and $\mathcal{Y}_{n, B}^{\prime}$ be as in the preceding lemma. Then:

(i) It is the case that $\lim _{\sup _{n \rightarrow \infty}} \nu\left(B_{n}\right)<1$.

(ii) For every subsequence of $\mathbb{N}$ such that $\nu\left(B_{n}\right)$ is bounded away from zero along the subsequence, we have along that subsequence that

$$
\lim _{n \rightarrow \infty}\left(\frac{\mathrm{Bal}_{n, v, b}\left(\mathcal{Y}_{n, B}^{\prime}\right)}{\mathrm{Bal}_{\nu, v, b}\left(B_{n}\right)}\right)=1 .
$$

Proof. (i) It is enough to prove that for every infinite subsequence $\mathcal{N}$ of $\mathbb{N}$ with $\inf _{n \in \mathcal{N}} \nu\left(B_{n}\right)>0$ we have $\limsup \nu\left(B_{n}\right)<1$ as $n \rightarrow \infty$ through $\mathcal{N}$. By (5.7) and (in the case $v=2$ ) (4.3) we have

$$
\frac{\operatorname{Vol}_{n, v}\left(\mathcal{Y}_{n}^{\prime}\right)-\operatorname{Vol}_{n, v}\left(\mathcal{Y}_{n}\right)}{\operatorname{Vol}_{n, v}\left(\mathcal{X}_{n}\right)} \rightarrow 0
$$

and since we assume $\operatorname{Vol}_{n, v}\left(\mathcal{Y}_{n}\right) \leq \operatorname{Vol}_{n, v}\left(\mathcal{X}_{n}\right) / 2$, by Lemma 4.3 we have for large enough $n$ that

$$
(2 / 3) \geq \frac{\operatorname{Vol}_{n, v}\left(\mathcal{Y}_{n}^{\prime}\right)}{\operatorname{Vol}_{n, v}\left(\mathcal{X}_{n}\right)} \geq \frac{\operatorname{Vol}_{n, v}\left(\mathcal{Y}_{n, B}^{\prime}\right)}{\operatorname{Vol}_{n, v}\left(\mathcal{X}_{n}\right)}=(1+o(1))\left(\frac{\int_{B_{n}} \rho(x)^{v} d x}{\int_{D} \rho(x)^{v} d x}\right),
$$


which gives us part (i).

(ii) Let $\mathcal{N} \subset \mathbb{N}$ be infinite with $\inf _{n \in \mathcal{N}} \nu\left(B_{n}\right)>0$. By Lemma 4.3 we have

$$
\lim _{n \rightarrow \infty, n \in \mathcal{N}}\left(\frac{\operatorname{Vol}_{n, v}\left(\mathcal{Y}_{n, B}^{\prime}\right) \operatorname{Vol}_{\nu, v}(D)}{\operatorname{Vol}_{n, v}\left(B_{n}\right) \operatorname{Vol}_{n, v}\left(\mathcal{X}_{n}\right)}\right)=1
$$

Set $W_{n}:=D \backslash B_{n}$ and $\mathcal{Y}_{n, W}^{\prime}:=\mathcal{Y}_{n}^{\prime} \cap W_{n}$. By part (i), $\nu\left(W_{n}\right)$ is bounded away from zero and therefore by applying Lemma 4.3 again, we have

$$
\lim _{n \rightarrow \infty, n \in \mathcal{N}}\left(\frac{\operatorname{Vol}_{n, v}\left(\mathcal{Y}_{n, W}^{\prime}\right) \operatorname{Vol}_{\nu, v}(D)}{\operatorname{Vol}_{n, v}\left(W_{n}\right) \operatorname{Vol}_{n, v}\left(\mathcal{X}_{n}\right)}\right)=1 .
$$

Moreover, since the number of white points in black boxes is at most $n \gamma_{n}$, using also (4.3) (in the case $v=2$ ) we have

$$
\frac{\operatorname{Vol}_{n, v}\left(\mathcal{X}_{n} \backslash \mathcal{Y}_{n, B}^{\prime}\right)-\operatorname{Vol}_{n, v}\left(\mathcal{Y}_{n, W}^{\prime}\right)}{\operatorname{Vol}_{n, v}\left(\mathcal{X}_{n}\right)}=\frac{\operatorname{Vol}_{n, v}\left(\left(\mathcal{X}_{n} \backslash \mathcal{Y}_{n}^{\prime}\right) \cap B_{n}\right)}{\operatorname{Vol}_{n, v}\left(\mathcal{X}_{n}\right)} \leq \text { const. } \times \gamma_{n}
$$

which tends to zero, and hence by (5.24) we have

$$
\lim _{n \rightarrow \infty, n \in \mathcal{N}}\left(\frac{\operatorname{Vol}_{n, v}\left(\mathcal{X}_{n} \backslash \mathcal{Y}_{n, B}^{\prime}\right) \operatorname{Vol}_{\nu, v}(D)}{\operatorname{Vol}_{n, v}\left(W_{n}\right) \operatorname{Vol}_{n, v}\left(\mathcal{X}_{n}\right)}\right)=1 .
$$

By using this, along with (5.23), we can obtain (5.22).

Lemma 5.7. Let $B_{n}$ and $\mathcal{Y}_{n, B}^{\prime}$ be as in the preceding lemma. For any subsequence of $\mathbb{N}$ there a further subsequence along which the functions $u_{n}$ converge in $L^{1}(\nu)$ to $\mathbf{1}_{A} / \mathrm{Bal}_{\nu, v, b}(A)$ for some $A \in \mathcal{B}(D)$ with $0<\nu(A)<1$.

Proof. We claim that it suffices to prove that

$$
\limsup _{n \rightarrow \infty}\left(\frac{\operatorname{Bal}_{n, v, b}\left(\mathcal{Y}_{n, B}^{\prime}\right)}{\operatorname{Bal}_{\nu, v, b}\left(B_{n}\right)}\right)<\infty
$$

Indeed, suppose (5.25) holds and for $n \in \mathbb{N}$ define the function $u_{n}:=\mathbf{1}_{B_{n}} / \mathrm{Bal}_{\nu, v, b}\left(B_{n}\right)$. Then by (5.25) and (5.17) the sequence $T V_{\phi, \tilde{r}_{n}}\left(u_{n}\right)$ is bounded, so we can apply Lemma 3.3 (iii), to deduce that for any subsequence of $\mathbb{N}$ there exists a further subsequence along which the functions $\mathbf{1}_{B_{n}} / \operatorname{Bal}_{\nu, v, b}\left(B_{n}\right)$ converge in $L^{1}(\nu)$ to a limiting function which must necessarily be of the form $\mathbf{1}_{A} / \operatorname{Bal}_{\nu, v, b}(A)$ with $A \in \mathcal{B}(D)$ and $0<\nu(A)<1$ (see Lemma 6 of [19]).

It remains to prove (5.25). First suppose $v=1$. By (3.12) we have for large enough $n$ that

$$
\left|\mathcal{Y}_{n, B}^{\prime}\right| \leq 2 n \nu\left(B_{n}\right)
$$


Also, by (3.12) and Lemma 5.6, for large enough $n$ we have

$$
\left|\mathcal{X}_{n} \backslash \mathcal{Y}_{n, B}^{\prime}\right| \leq 2 n \nu\left(W_{n}\right)+\gamma_{n} n \nu\left(B_{n}\right) \leq 3 n \nu\left(W_{n}\right)
$$

and combined with (5.26) this gives us (5.25) in the case $v=1$ (either for $b=1$ or $b=2)$.

Now suppose $v=2$. Set $V_{n}:=\operatorname{Vol}_{n, 2}\left(\mathcal{X}_{n}\right)$. Using (5.26) and (4.3) we have that

$$
V_{n}^{-1} \operatorname{Vol}_{n, 2}\left(\mathcal{Y}_{n, B}^{\prime}\right) \leq \text { const. } \times \nu\left(B_{n}\right) .
$$

Since $\nu\left(B_{n}\right)$ is bounded away from 1 by Lemma 5.6 (i), using (4.3) and (3.12) we have

$$
\limsup \left(\frac{\operatorname{Vol}_{n, 2}\left(\mathcal{X}_{n} \backslash \mathcal{Y}_{n}^{\prime}\right)}{\nu\left(W_{n}\right) V_{n}}\right)<\infty
$$

and combined with (5.27) this gives us (5.25) for $v=2$.

Proof of Theorem 2.1. By Lemma 5.7, for any subsequence of $\mathbb{N}$ there exists a further subsequence along which the functions $\mathbf{1}_{B_{n}} / \mathrm{Bal}_{\nu, v, b}\left(B_{n}\right)$ converge in $L^{1}(\nu)$ to a limiting function of the form $\mathbf{1}_{A} / \mathrm{Bal}_{\nu, v, b}(A)$ with $A \in \mathcal{B}(D)$ and $0<\nu(A)<1$. Then $\nu\left(B_{n}\right)$ is bounded away from zero.

Then by part (i) of Lemma 3.3, and Lemma 5.6, we have along this subsequence that

$$
\begin{array}{r}
\sigma_{\phi} T V\left(\mathbf{1}_{A} / \operatorname{Bal}_{\nu, v, b}(A)\right) \leq \liminf _{n \rightarrow \infty} T V_{\phi, \tilde{r}_{n}}\left(\mathbf{1}_{B_{n}} / \operatorname{Bal}_{\nu, v, b}\left(B_{n}\right)\right) \\
\leq \liminf _{n \rightarrow \infty} T V_{\phi, \tilde{r}_{n}}\left(\mathbf{1}_{B_{n}} / \mathrm{Bal}_{n, v, b}\left(\mathcal{Y}_{n, B}^{\prime}\right)\right) .
\end{array}
$$

Therefore by (5.16), followed by (4.1), we have along this subsequence that

$$
\begin{array}{r}
\left(\sigma_{\phi} / 2\right) T V\left(\mathbf{1}_{A} / \operatorname{Bal}_{\nu, v, b}(A)\right) \leq \liminf _{n \rightarrow \infty}\left(\frac{\mathrm{CHE}_{v, b}\left(G_{\phi}\left(\mathcal{X}_{n}, r_{n}\right)\right)}{n^{2} r_{n}^{d+1}}\right) \\
\leq \limsup _{n \rightarrow \infty}\left(\frac{\mathrm{CHE}_{v, b}\left(G_{\phi}\left(\mathcal{X}_{n}, r_{n}\right)\right)}{n^{2} r_{n}^{d+1}}\right) \leq \mathrm{CHE}_{v, b}(D, \rho) \sigma_{\phi} / 2 .
\end{array}
$$

By the definition (2.5) the inequalities in (5.28) are all equalities and the set $A$ is a minimiser in (2.5). This gives us the asserted convergence (2.10).

Proof of Theorem 2.2. To prove this we re-examine the preceding proof. For each $n \in \mathbb{N}$, let $\mathcal{Y}_{n} \subset \mathcal{X}_{n}$ be a minimiser as in the definition (2.3) of $\mathrm{CHE}_{v, b}\left(G_{\phi}\left(\mathcal{X}_{n}, r_{n}\right)\right)$ with $\operatorname{Vol}_{n, v}\left(\mathcal{Y}_{n}\right) / \operatorname{Vol}_{n, v}\left(\mathcal{X}_{n}\right) \leq 1 / 2$, as before.

Let $\mathcal{Y}_{n}^{\prime}$ and $B_{n}$ be as in the previous proof. As shown there, for every subsequence there is a further subsequence along which $\mathbf{1}_{B_{n}} \rightarrow \mathbf{1}_{A}$ in $L^{1}(\nu)$ for some optimising set $A$. 
Let $\mu_{n}:=\sum_{y \in \mathcal{Y}_{n}} n^{-1} \delta_{y}$ and let $\mu_{n}^{\prime}:=\sum_{y \in \mathcal{Y}_{n}^{\prime}} n^{-1} \delta_{y}$. To demonstrate (2.12), we need to show that the sequence $\left(\mu_{n}\right)$ of measures converges weakly to the restriction of $\nu$ to $A$. By the Portmanteau theorem [5], it is enough to show that for any uniformly continuous function $f$ on $D$ we have $\mu_{n}(f) \rightarrow \nu\left(f \mathbf{1}_{A}\right)$. Since any such $f$ is bounded, we have by (5.7) that $n^{-1}\left|\mu_{n}^{\prime}(f)-\mu_{n}(f)\right| \rightarrow 0$.

On $W_{n}:=D \backslash B_{n}$ the density of points relative to the measure $n \nu$ is at most $\gamma_{n}$; that is, $\mu_{n}^{\prime}\left(W_{n}\right) \leq \gamma_{n} \nu(D)$ which tends to zero.

Since $f$ is uniformly continuous, given $\varepsilon>0$ we can find $n_{0}$ such that for $n \geq n_{0}$ we have for all $i \in S_{n}$ that $\bar{f}_{i, n}:=\sup _{Q_{i, n}} f$ and $\underline{f}_{i, n}:=\inf _{Q_{i, n}} f$ satisfy $\bar{f}_{i, n}-\underline{f}_{i, n}<\varepsilon$. Then for $n \geq n_{0}$, setting $f_{\max }=\sup _{x \in D} f(x)$, we have

$$
\begin{array}{r}
\mu_{n}^{\prime}(f) \leq\left(\sum_{\left\{i: Q_{i, n} \subset B_{n}\right\}}\left(1+\gamma_{n}\right) \nu\left(Q_{i, n}\right) \bar{f}_{i, n}\right)+f_{\max } \mu_{n}^{\prime}\left(W_{n}\right) \\
\leq\left(1+\gamma_{n}\right) \int_{A}(f(x)+\varepsilon) \nu(d x)+o(1),
\end{array}
$$

and therefore

$$
\limsup _{n \rightarrow \infty} \mu_{n}^{\prime}(f) \leq \int_{B}(f(x)+\varepsilon) \nu(d x)
$$

Also,

$$
\mu_{n}^{\prime}(f) \geq \sum_{\left\{i: Q_{i, n} \subset B_{n}\right\}}\left(1-2 \gamma_{n}\right) \nu\left(Q_{i, n}\right) \underline{f}_{i, n} \geq\left(1-2 \gamma_{n}\right) \int_{B_{n}}(f(x)-\varepsilon) \nu(d x)
$$

so that

$$
\liminf _{n \rightarrow \infty} \mu_{n}^{\prime}(f) \geq \int_{A}(f(x)-\varepsilon) \nu(d x) .
$$

Combining this with (5.29) gives us (2.12).

Proof of Corollary 2.3. Assume that the hypotheses of Theorem 2.1 apply, and also that the minimising set $A$ in the definition (2.5) of $\mathrm{CHE}_{v, b}(D, \rho)$ is unique, up to complementation and adding or removing sets of $(d-1)$-dimensional measure zero. We shall use the following. Set $\nu_{n}:=\sum_{y \in \mathcal{X}_{n}} n^{-1} \delta_{y}$. Given any uniformly continuous function $f$ on $D$, similarly to (5.29) and (5.30) it can be shown that

$$
\nu_{n}(f) \rightarrow \nu(f) .
$$

We also use the fact that the topology of weak convergence of probability measures on $D$ is metrizable by the Prohorov metric (here denoted $d_{\pi}$ ) on the space of such measures. See [5, page 72], where a definition of this metric can also be found. 
Let $\mathcal{Y}_{n}$ be a sequence of minimisers in the definition (2.3) of $\mathrm{CHE}_{v, b}\left(G_{\phi}\left(\mathcal{X}_{n}, r_{n}\right)\right)$. Then we claim that

$$
\min \left(d_{\pi}\left(\mu_{n},\left.\nu\right|_{A}\right), d_{\pi}\left(\mu_{n},\left.\nu\right|_{A^{c}}\right)\right) \rightarrow 0
$$

It is straightforward to deduce this from the part of Theorem 2.2 already proved, along with our uniqueness assumption regarding $A$, noting also that if we add or remove a Lebesgue-null set to/from $A$, the measure $\left.\nu\right|_{A}$ is unchanged. Now take

$$
j(n)=\left\{\begin{array}{l}
1 \text { if } d\left(\mu_{n},\left.\nu\right|_{A}\right) \leq d\left(\mu_{n},\left.\nu\right|_{A^{c}}\right) \\
0 \text { otherwise }
\end{array}\right.
$$

On the sequence of $n$ for which $j(n)=1$ (if this sequence is infinite), by (5.32) we have $d_{\pi}\left(\mu_{n},\left.\nu\right|_{A}\right) \rightarrow 0$ so $\mu_{n}$ converges weakly to $\left.\nu\right|_{A}$.

On the sequence of $n$ for which $j(n)=0$ (if this sequence is infinite), by (5.32) we have $d_{\pi}\left(\mu_{n},\left.\nu\right|_{A^{c}}\right) \rightarrow 0$ so $\mu_{n}$ converges weakly to $\left.\nu\right|_{A^{c}}$. Then using (5.31), we have for any uniformly continuous function $f$ on $D$ that $\left.\left(\nu_{n}-\mu_{n}\right)(f) \rightarrow \nu\right|_{A}(f)$, so $\nu_{n}-\mu_{n}$ converges weakly to $\left.\nu\right|_{A}$.

Putting the last two paragraphs together gives us the desired conclusion.

\section{The bisection problem}

In this section we prove Theorem 2.4. The result is immediate from Lemmas 6.1 and 6.2. We assume throughout this section that the assumptions of Theorem 2.4 apply.

Lemma 6.1. It is the case that

$$
\limsup _{n \rightarrow \infty}\left(\frac{\operatorname{MBIS}\left(G_{\phi}\left(\mathcal{X}_{n}, r_{n}\right)\right)}{n^{2} r_{n}^{d+1}}\right) \leq\left(\sigma_{\phi} / 2\right) \operatorname{MBIS}_{\nu}(D), \quad \text { a.s. }
$$

Proof. Let $A \in \mathcal{B}(D)$ with $\nu(A)=1 / 2$. Set $\mathcal{Y}_{n}=\mathcal{X}_{n} \cap A$. Then by (4.13),

$$
\left(n^{2} r_{n}^{d+1}\right)^{-1} \operatorname{Cut}_{n, \phi}\left(\mathcal{Y}_{n}\right) \rightarrow\left(\sigma_{\phi} / 2\right) T V\left(\mathbf{1}_{A}\right) .
$$

Also $n^{-1}\left|\mathcal{Y}_{n}\right| \rightarrow 1 / 2$ by the strong law of large numbers, but of course this does not tell us that $\left|\mathcal{Y}_{n}\right|=\lfloor n / 2\rfloor$. Set $M_{n}:=\left|\mathcal{Y}_{n}\right|-\lfloor n / 2\rfloor$. Using the Chernoff bounds (3.10) and (3.11), Taylor's Theorem (as in the proof of Lemma 3.2) and the Borel-Cantelli lemma, we have almost surely that for large enough $n$,

$$
\left|M_{n}\right| \leq 3(n \log n)^{1 / 2}
$$


As in the preceding section, we shall say that points in $\mathcal{Y}_{n}$ are black and points in $\mathcal{X}_{n} \backslash \mathcal{Y}_{n}$ are white. If $M_{n}<0$ let us pick $\left|M_{n}\right|$ points in $\mathcal{X}_{n} \backslash \mathcal{Y}_{n}$, and add them to $\mathcal{Y}_{n}$ (i.e., change their colour from white to black). If $M_{n}>0$, pick $M_{n}$ points in $\mathcal{Y}_{n}$, and remove them from $\mathcal{Y}_{n}$ (i.e., change their colour from black to white). In both cases let $\mathcal{Y}_{n}^{\prime}$ be the resulting set of black points. Then $\left|\mathcal{Y}_{n}^{\prime}\right|=\lfloor n / 2\rfloor$.

By (4.3), the total weight of cut edges created or destroyed by changing from $\mathcal{Y}$ to $\mathcal{Y}^{\prime}$ is at most a constant times $n r_{n}^{d}\left|M_{n}\right|$. Therefore using (6.3) we have

$$
\begin{array}{r}
\left(n^{2} r_{n}^{d+1}\right)^{-1}\left|\operatorname{Cut}_{n, \phi}\left(\mathcal{Y}_{n}^{\prime}\right)-\operatorname{Cut}_{n, \phi}\left(\mathcal{Y}_{n}\right)\right|=O\left(\left(n^{-1} \log n\right)^{1 / 2} r_{n}^{-1}\right) \\
=O\left(\left(n r_{n}^{d} / \log n\right)^{-1 / 2} r_{n}^{(d-2) / 2}\right)
\end{array}
$$

which tends to zero by the assumptions $n r_{n}^{d} \gg \log n$ and $d \geq 2$. Combined with (6.2) this shows that

$$
\limsup _{n \rightarrow \infty}\left(\frac{\operatorname{MBIS}\left(G_{\phi}\left(\mathcal{X}_{n}, r_{n}\right)\right)}{n^{2} r_{n}^{d+1}}\right) \leq \limsup _{n \rightarrow \infty}\left(\frac{\operatorname{Cut}_{n, \phi}\left(\mathcal{Y}_{n}^{\prime}\right)}{n^{2} r_{n}^{d+1}}\right)=\left(\sigma_{\phi} / 2\right) T V\left(\mathbf{1}_{A}\right) .
$$

Taking the infimum over all $A$ and using (2.16), this gives us (6.1).

Lemma 6.2. It is the case that

$$
\liminf _{n \rightarrow \infty}\left(\frac{\operatorname{MBIS}\left(G_{\phi}\left(\mathcal{X}_{n}, r_{n}\right)\right)}{n^{2} r_{n}^{d+1}}\right) \geq\left(\sigma_{\phi} / 2\right) \operatorname{MBIS}_{\nu}(D), \quad \text { a.s. }
$$

Proof. We argue similarly to the proof in Section 5. For each $n \in \mathbb{N}$ let $\mathcal{Y}_{n}$ be a bisection of $\mathcal{X}_{n}$ (i.e. a subset with $\lfloor n / 2\rfloor$ elements) that achieves the minimum in the definition (2.14). Define the boxes $Q_{i, n}$ as in Section 5, and define black, grey and white boxes as we did there. By Lemma [5.3, there is a set $\mathcal{Y}_{n}^{\prime}$ satisfying (5.7) and inducing no grey boxes, such that

$$
\operatorname{MBIS}\left(G_{\phi}\left(\mathcal{X}_{n}, r_{n}\right)\right)=\operatorname{Cut}_{n, \phi}\left(\mathcal{Y}_{n}\right) \geq \operatorname{Cut}_{n, \phi}^{\prime}\left(\mathcal{Y}_{n}^{\prime}\right) \geq Z_{n}
$$

where $Z_{n}$ denotes the contribution to $\mathrm{Cut}_{n, \phi}^{\prime}\left(\mathcal{Y}_{n}^{\prime}\right)$ from edges with exactly one endpoint in $B_{n}$, and $B_{n}$ denotes the union of the black boxes induced by $\mathcal{Y}_{n}^{\prime}$. Then by (5.14),

$$
\limsup _{n \rightarrow \infty}\left(\frac{\operatorname{MBIS}\left(G_{\phi}\left(\mathcal{X}_{n}, r_{n}\right)\right)}{n^{2} r_{n}^{d+1}}\right) \geq(1 / 2) \limsup _{n \rightarrow \infty} T V_{\phi, \tilde{r}_{n}}\left(\mathbf{1}_{B_{n}}\right),
$$

and the left side of (6.5) is finite by Lemma 6.1. By the compactness property (Lemma 3.3 (iii)), for any infinite subsequence $\mathcal{N} \subset \mathbb{N}$, we may find an infinite subsequence $\mathcal{N}^{\prime} \subset \mathcal{N}$ such that as $n \rightarrow \infty$ through $\mathcal{N}^{\prime}$, the fucntions $\mathbf{1}_{B_{n}}$ converge in $L^{1}(\nu)$ to a limit, necessarily of the form $\mathbf{1}_{B}$ for some $B \in \mathcal{B}(D)$. 
Using (5.7) and the fact that the original $\mathcal{Y}_{n}$ was a bisection, we have that $n^{-1}\left|\mathcal{Y}_{n}^{\prime}\right| \rightarrow 1 / 2$. Then using (3.12), (3.13) and the fact that we take $\gamma_{n} \rightarrow 0$ so the proportion of black vertices in white boxes or white vertices in black boxes vanishes, we have that $\nu\left(B_{n}\right) \rightarrow 1 / 2$. Therefore $\nu(B)=1 / 2$.

By the definition in (2.16), and the liminf lower bound from Lemma 3.3 (i), we have as $n \rightarrow \infty$ through $\mathcal{N}^{\prime}$ that

$$
\left(\sigma_{\phi} / 2\right) \operatorname{MBIS}_{\nu}(D) \leq\left(\sigma_{\phi} / 2\right) T V\left(\mathbf{1}_{B}\right) \leq \liminf _{n \rightarrow \infty}(1 / 2) T V_{\phi, \tilde{r}_{n}}\left(\mathbf{1}_{B_{n}}\right),
$$

and since (6.5) still holds with lim sup replaced by liminf on both sides, we thus obtain (6.4).

Acknowledgement. We thank Nicolás García Trillos and Ery Arias Castro for answering some questions regarding their work on this subject.

The research leading to this paper was partially carried out during an extended visit of the second author to Utrecht University. He thanks the Department of Mathematics at Utrecht University for its hospitality.

\section{References}

[1] Arias-Castro, E., Pelletier, B. and Pudlo, P. (2012). The normalized graph cut and Cheeger constant: from discrete to continuous. Adv. Appl. Probab. 44, 907937.

[2] Benjamini, I. and Mossel, E. (2003). On the mixing time of a simple random walk on the super critical percolation cluster. Probab. Theory Relat. Fields 125, 408-420.

[3] Beardwood, J., Halton, J.H. and Hammersley, J. M. (1959). The shortest path through many points. Proc. Cambridge Philos. Soc. 55, 299-327.

[4] Bhatt, S.N. and Leighton, F.T. (1984). A framework for solving VLSI graph layout problems. J. Comput. System Sci. 28, 300-343.

[5] Billingsley, P. (1999). Convergence of Probability Measures. 2nd ed., Willey.

[6] Braides, A. (1998). Approximation of Free-Discontinuity Problems. Springer, Berlin.

[7] Buser, P. (1982). A note on the isoperimetric constant. Ann. Sci. École Norm. Sup. (4) 15, 213-230. 
[8] Carlsson, G. (2009). Topology and data. Bull. Amer. Math. Soc. (N.S.) 46, $255-308$.

[9] Caselles, V., Chambolle, A. and Novaga, M. (2010) Some remarks on uniqueness and regularity of Cheeger sets. Rend. Semin. Mat. Univ. Padova 123, 191-201.

[10] Cheeger, J. (1970). A lower bound for the smallest eigenvalue of the Laplacian. Problems in analysis (Papers dedicated to Salomon Bochner, 1969), pp. 195-199. Princeton Univ. Press, Princeton, N.J.

[11] Chung, F.R.K. (1997) Spectral Graph Theory. American Mathematical Society, Providence, RI.

[12] Cuevas, A., Fraiman, R. and Rodrguez-Casal, A. (2007). A nonparametric approach to the estimation of lengths and surface areas. Ann. Statist. 35, 1031-051.

[13] Díaz, J., Penrose, M.D., Petit, J. and Serna, M. (2001). Approximating layout problems on random geometric graphs. J. Algorithms 39, 78-116.

[14] Diekmann, R., Monien, B., Preis, R. (1995). Using helpful sets to improve graph bisections. Interconnection networks and mapping and scheduling parallel computations (D.F. Hsu, A.L. Rosenberg and D. Sotteau, eds.) 57-73, Amer. Math. Soc., Providence, RI,

[15] Edelsbrunner, H. and Harer, J.L. (2010). Computational topology. An introduction. American Mathematical Society, Providence, RI.

[16] Garca Trillos, N. and Slepcev, D. (2015). On the rate of convergence of empirical measures in $\infty$-transportation distance. Canad. J. Math. 67, 1358-1383.

[17] García Trillos, N. and Slepčev, D. (2016). Continuum limit of total variation on point clouds. Arch. Ration. Mech. Anal. 220, 193-241.

[18] García Trillos, N., Slepčev, D. and von Brecht, J. (2017). Estimating perimeter using graph cuts. Adv. in Appl. Probab. 49, 1067-1090.

[19] García Trillos, N., Slepčev, D., von Brecht, J., Laurent, T. and Bresson, X. (2016). Consistency of Cheeger and ratio graph cuts. J. Mach. Learn. Res. 17 (181), 1-46.

[20] Henrot, A. and Pierre, M. (2005) Variation et Optimisation de Formes. Une Analyse Géométrique. Springer, Berlin. 
[21] Kahle, M. (2014). Topology of random simplicial complexes: a survey. Algebraic topology: applications and new directions, 201-221, Contemp. Math., 620, Amer. Math. Soc., Providence, RI,

[22] Kiwi, M. and Mitsche, D. (2018) Spectral gap of random hyperbolic graphs and related parameters. Ann. Appl. Probab. 28, 941-989.

[23] Leighton, T., Shor, P. (1989) Tight bounds for minimax grid matching with applications to the average case analysis of algorithms. Combinatorica 9, 161187.

[24] Penrose, M. (2003) Random Geometric Graphs. Oxford University Press.

[25] Penrose, M.D. (1997). The longest edge of the random minimal spanning tree. Ann. Appl. Probab. 7, 340-361.

[26] Penrose, M.D. (2000) Vertex ordering and partitioning problems for random spatial graphs. Ann. Appl. Probab. 10, 517-538.

[27] Shor, P. W. and Yukich, J. E. (1991). Minimax grid matching and empirical measures. Ann. Probab. 19, 1338-1348.

[28] von Luxburg, U. (2007) A tutorial on spectral clustering. Stat. Comput. 17 $395-416$. 